\title{
Hedgehog signaling induces PD-L1 expression and tumor cell proliferation in gastric cancer
}

\author{
Jayati Chakrabarti ${ }^{1}$, Loryn Holokai ${ }^{2}$, LiJyun Syu ${ }^{3}$, Nina G. Steele ${ }^{4}$, Julie Chang ${ }^{5}$, Jiang \\ Wang ${ }^{6}$, Syed Ahmed ${ }^{7}$, Andrzej Dlugosz ${ }^{3,8}$ and Yana Zavros ${ }^{1}$ \\ ${ }^{1}$ Department of Pharmacology and Systems Physiology, University of Cincinnati, Cincinnati, OH, USA \\ ${ }^{2}$ Department of Molecular Genetics, Biochemistry and Microbiology, University of Cincinnati, OH, USA \\ ${ }^{3}$ Department of Dermatology, University of Michigan, Ann Arbor, MI, USA \\ ${ }^{4}$ Division of Developmental Biology, University of Michigan, Ann Arbor, MI, USA \\ ${ }^{5}$ Department of Biomedical Engineering, University of Cincinnati, Cincinnati, OH, USA \\ ${ }^{6}$ Department of Pathology and Laboratory Medicine, University of Cincinnati College of Medicine, Cincinnati, OH, USA \\ ${ }^{7}$ Department of Surgery, University of Cincinnati Cancer Institute, Cincinnati, OH, USA \\ ${ }^{8}$ Department of Cell and Developmental Biology, University of Michigan, Ann Arbor, MI, USA \\ Correspondence to: Yana Zavros, email: yana.zavros@uc.edu \\ Keywords: gastric cancer organoids, PD-1; cytotoxic T lymphocytes; dendritic cells \\ Received: August 29, $2018 \quad$ Accepted: November 26, $2018 \quad$ Published: December 21, 2018
}

Copyright: Chakrabarti et al. This is an open-access article distributed under the terms of the Creative Commons Attribution License 3.0 (CC BY 3.0), which permits unrestricted use, distribution, and reproduction in any medium, provided the original author and source are credited.

\section{ABSTRACT}

Tumor cells expressing programmed cell death ligand 1 (PD-L1) interact with PD-1 on CD8+ cytotoxic T lymphocytes (CTLs) to inhibit CTL effector function. In gastric cancer, the mechanism regulating PD-L1 is unclear. The Hedgehog (Hh) signaling pathway is reactivated in various cancers including gastric. Here we tested the hypothesis that Hh-induced PD-L1 inactivates effector $\mathrm{T}$ cell function and allows gastric cancer cell proliferation. Mouse organoids were generated from tumors of a triple-transgenic mouse model engineered to express an activated GLI2 allele, GLI2A, in Lgr5-expressing stem cells, (mTGOs) or normal mouse stomachs (mGOs). Bone marrow-derived dendritic cells (DCs) were pulsed with conditioned media collected from normal $\left(\mathrm{mGO}^{\mathrm{cm}}\right)$ or cancer $\left(\mathrm{mTGO}^{\mathrm{cm}}\right)$ organoids. Pulsed DCs and CTLs were then co-cultured with either mGOs or mTGOs in the presence of PD-L1 neutralizing antibody (PD-L1Ab). Human-derived gastric cancer organoids (huTGOs) were used in drug and xenograft assays. Hh/Gli inhibitor, GANT-61 significantly reduced the expression of PD-L1 and tumor cell proliferation both in vivo and in vitro. PD-L1Ab treatment induced tumor cell apoptosis in $\mathrm{mTGO} / \mathrm{immune}$ cell co-cultures. GANT-61 treatment sensitized huTGOs to standard-of-care chemotherapeutic drugs both in vivo and in vitro. Thus, Hh signaling mediates PD-L1 expression in gastric cancer cells and subsequently promotes tumor proliferation.

\section{INTRODUCTION}

The effective diagnosis and treatment of the major gastric cancer risk factor Helicobacter pylori (H. pylori) has resulted in the decreased incidence of gastric cancer in the United States $[1,2]$. However, the incidence of gastric cancer varies throughout the world, with highrisk areas including East Asia (China and Japan), Eastern Europe, and Central and South America [2, 3]. The disease becomes symptomatic in the advanced stages, and the 5-year survival rate for patients diagnosed with this malignancy is only $10 \%-30 \%[1,2,4]$. Given the poor response of gastric cancer to various existing treatment modalities, there is a need for approaches to predict individual therapy responses [1].

Despite the advances of targeted therapy using trastuzumab for HER2-positive gastro-esophageal cancers, anti-VEGFR2 monoclonal antibody ramucirumab and paclitaxel that improve survival, patients with metastatic gastro-esophageal cancer live for less than 2 years $[5,6]$. 
Immune-checkpoint blockade with anti-CTLA4, anti-PD-1 and anti-PD-L1 antibodies has advanced the treatment of many cancers including gastric adenocarcinomas [7]. Programmed death-1 (PD-1) and programmed death ligand-1 (PD-L1) are two- immune-checkpoint molecules for targeted cancer therapy. Tumor cells expressing PDL1 interact with PD-1 on CD8+ cytotoxic T lymphocytes (CTLs). This interaction inhibits CTL effector function, subsequently leading to immune evasion and cancer cell proliferation [8-10]. PD-L1+ $(\mathrm{B} 7-\mathrm{H} 1+)$ gastric cancer stem cells exhibit an increased proliferative capacity [11]. While clinical trials using immune-checkpoint inhibition is proven to be promising for the treatment of gastric cancer, there are no established selection criteria to predict whether a patient will benefit from immunotherapy alone or with combination therapy.

Hedgehog $(\mathrm{Hh})$ signaling plays a crucial role in growth and morphogenesis in a wide variety of tissues during embryonic development [12]. Importantly, the $\mathrm{Hh}$ signaling pathway is often overexpressed in various cancers including gastric and pancreatic (reviewed in [13]). Based on the TCGA data, we find that Gli2, Shh, Ptch1, Ptch2, Smo, are altered in 7\%, 6\%, 10\%, 7\% and $8 \%$ of 258 patients selected for the study, respectively [14]. Importantly, studies suggest that Hh signaling is one of regulatory pathways of PD-L1 expression and that inhibiting Hh signaling may induce lymphocyte antitumor activity [15]. Thus, there is interest in targeting the Hh pathway as a potential therapeutic target for the treatment of these cancers. In the current study, we sought to investigate the role of $\mathrm{Hh}$ signaling as a mediator of PD-L1 expression during gastric tumorigenesis using an in vivo mouse model of gastric cancer, in vitro mousederived gastric cancer organoid/immune cell co-culture, and human-derived gastric cancer organoid drug assays.

\section{RESULTS}

\section{Inhibition of Hh signaling results in a decreased PD-L1 expression that correlates with loss of tumor formation in $i L g r 5 ; G L I 2 A$ mice}

To identify whether there was a correlation between induced Hh signaling within the gastric epithelium and induction of PD-L1 expression in vivo, expression of PDL1 was measured in $i \operatorname{Lgr} 5 ; G L I 2 A$ mice treated with $\mathrm{Hh} /$ Gli inhibitor GANT61 (Figure 1). As documented in the original report, activation of GLI2A in Lgr5+ gastric stem cells led to the rapid development of gastric tumors in the antrum after 3 weeks of doxycycline and vehicle treatment (Figure 1B) compared to control treated mice (Figure 1A) [16]. Unlike vehicle treated mice (Figure 1B), iLgr5; GLI2A GANT61 blocked the development of adenocarcinoma (Figure 1C). In $i \operatorname{Lgr} 5$; GLI2A mice, within the tumor region Gli 2 (green) was clearly expressed (Figure 1D). Although Gli2 was highly expressed within the IF-positive chief cells of the corpus/fundus of $i \operatorname{Lgr} 5 ; G L I 2 A$ mice, tumors did not develop in this region of the stomach (Figure 1E). Consistent with studies by Leushacke et al. [17], these data would indeed show the induction of Gli2 in Lgr5+ cells within a subset of chief cells in the stomach. However, overexpression of Gli2 in the chief cells does not contribute to tumorigenesis in this region of the stomach, but rather may be important in epithelial regeneration in response to injury as demonstrated by our group $[18,19]$.

Immunofluorescence staining for PD-L1 revealed increased expression within gastric adenocarcinoma of vehicle treated $i \operatorname{Lgr} 5 ; G L I 2 A$ mice (Figure 1G, 1I) compared to controls (Figure 1F). PD-L1 expression localized within metaplastic cells that were positive for gastric cancer marker CD44v9 (Figure 1J). The expression of PD-L1 was significantly decreased in the stomachs of mice treated with GANT61 (Figure 1H), a response that was confirmed by qRT-PCR (Figure 1K).

In canonical Hh signaling, Hh ligands bind to the receptor Patched $(\mathrm{PTCH})$ that leads to the activation of key pathway activator Smoothened (Smo), followed by activation of the GLI transcription factors. As a consequence of Smo activation, transcription of Gli target genes including Gli1, PTCH and HHIP are upregulated when the signal is triggered [20]. Decreased PD-L1 expression in response to GANT61 correlated with decreased expression of Hh canonical signaling genes, PTCH1, PTCH2, GLI1 and HHIP (Figure 1L).

Immunohistochemical staining for proliferative marker proliferating cell nuclear antigen (PCNA) revealed increased proliferating cells within the tumors of iLgr 5; GLI2A mice (Figure 2B, 2D) compared to control animals (Figure 2A, 2D). ILgr5;GLI2A mice treated with GANT61 had reduced proliferation wtihin the gastric epithelium (Figure 2C, 2D). Immunohistochemical staining showed increased CD8+ cytotoxic $\mathrm{T}$ cells infiltrating within GANT61-treated tumors (Figure 2G) compared to control stomachs (Figure 2F). In support of the immunohistochemistry, qRT-PCR data also demonstrated higher expression of CD8 as well as Granzyme B in GANT61 treated mice (Figure 2E). Collectively, these data confirm the previous report demonstrating that activation of GLI2A in Lgr5+ gastric stem cells leads to the development of gastric adenocarcinoma [16]. In addition, with the development of gastric cancer in response to Hh signaling is the robust expression of PD-L1 within tumor tissue.

\section{Inhibition of Hh signaling results in decreased PD-L1 expression and tumor cell proliferation in $i L g r 5 ; G L I 2 A$ mouse-derived gastric cancer organoids}

To further identify the role of Hh signaling in the regulation of $\mathrm{PD}-\mathrm{L} 1$ within gastric cancer cells, we used an organoid model derived from the tumor tissue 
of $i \operatorname{Lgr} 5$; GLI2A mice (mTGOs). Compared to organoids derived from the stomachs of normal control animals (mFGOs) (Figure 3A-3D), mTGOs were not only significantly more proliferative (mFGO $3.17 \pm 1.35 \%$
EdU/Total Cell Number, mTGO $43.9 \pm 10.7 \%$ EdU/Total Cell Number), but also expressed increased Gli2 and PDL1 expression (Figure 3E-3H). Tumor-derived mTGOs treated with GANT61 showed decreased expression
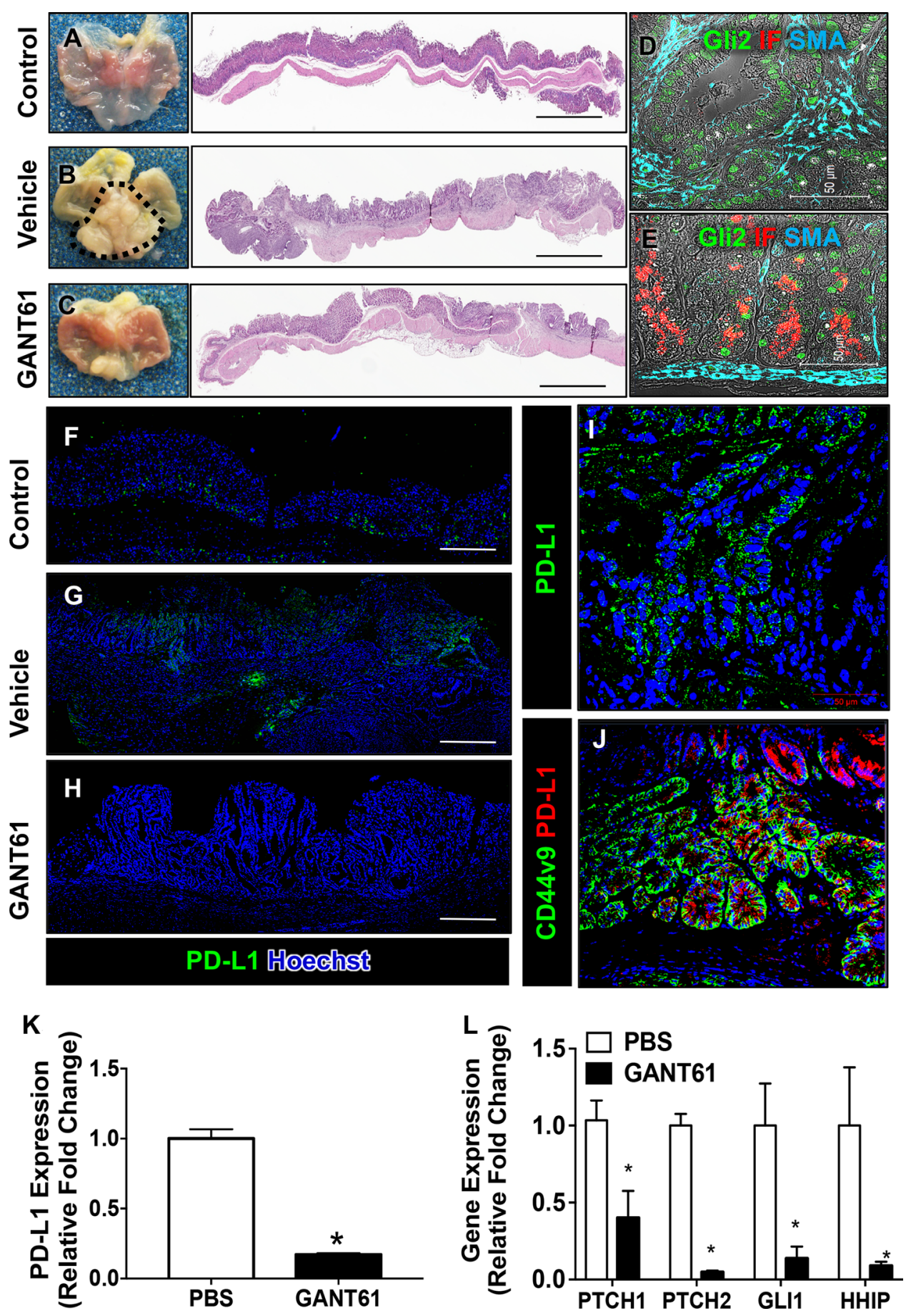

Figure 1: Histological changes and PD-L1 expression in iLgr5;GLI2A mice treated with GANT61. H\&E staining of sections collected from (A) control, (B) vehicle treated, and (C) GANT61 treated $i \operatorname{Lgr} 5 ; G L I 2 A$ mice. Immunofluorescence staining for Gli2 (green), intrinsic factor (IF, red) and smooth muscle actin (SMA, cyan) from sections collected from vehicle treated $i L g r 5 ; G L I 2 A$ mice in (D) fundus, and (E) antral tumor stomach regions. Immunofluorescence staining for PD-L1 expression (green) from sections collected from $(\mathbf{F})$ control, $(\mathbf{G})$ vehicle treated, and $(\mathbf{H})$ GANT61 treated $i L g r 5 ; G L I 2 A$ mice. (I) Shows a higher magnification of $(\mathbf{G})$. (J) Immunofluorescence of CD44v9 (green) and PD-L1 (red) in tumor tissue. Quantitative RT-PCR analysis for expression of (K) PD-L1 and (L) the downstream Hh canonical signaling genes, PTCH1, PTCH2, GLI1 and HHIP. ${ }^{*} P<0.05$ compared to PBS/vehicle control group, $n=5$ per group. 
of not only PD-L1 (Figure 3I), but also proliferating EdU-positive cells (Figure 3I, 3J) at concentration of 5 and $10 \mu \mathrm{M}$ of the inhibitor compared to the vehicle control $(0 \mu \mathrm{M})$. Western blots showed significantly reduced expression of $\mathrm{PD}-\mathrm{L} 1$ in response to 5 and $10 \mu \mathrm{M}$ GANT61 treated mTGOs (Figure 3K, 3L). Our in vitro data support the role of $\mathrm{Hh}$ signaling as a regulator of cancer cell proliferation and PD-L1 expression.
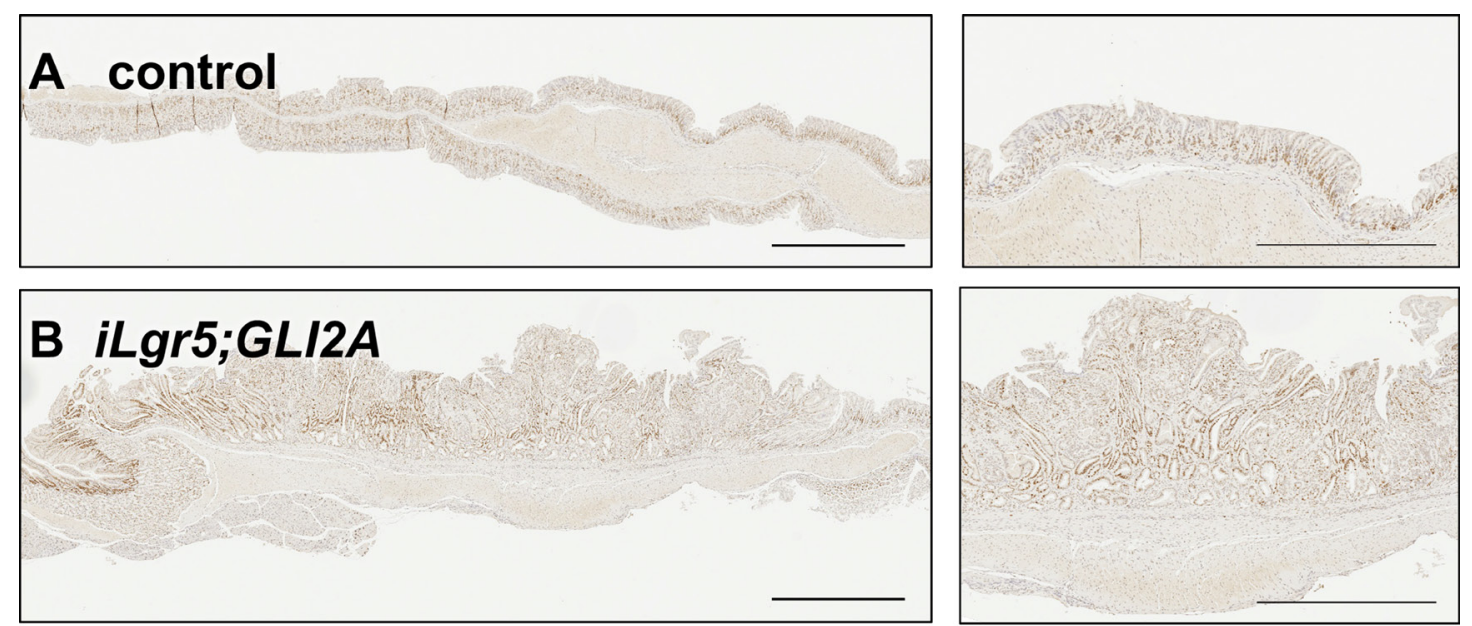

\section{C iLgr5;GLI2A + GANT61}
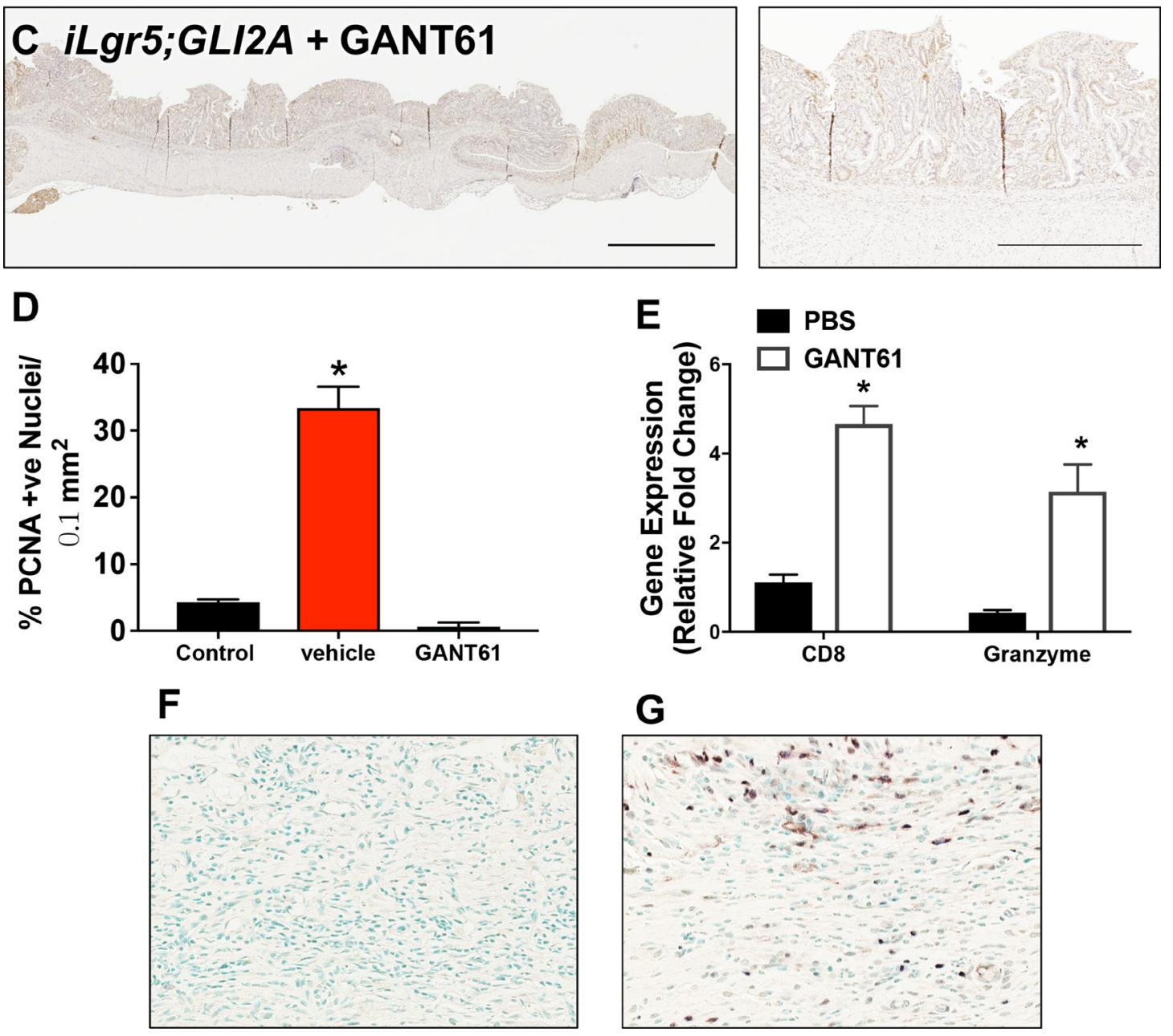

Figure 2: Changes in epithelial and tumor cell proliferation in iLgr5;GLI2A mice treated with GANT61. Immunohistochemical staining for PCNA expression (brown nuclei) of sections collected from (A) control, (B) vehicle treated, and (C) GANT61 treated $i L g r 5$; GLI2A mice. (D) Percentage of PCNA positive nuclei per $0.1 \mathrm{~mm}^{2}$ section was quantified. (E) Quantitative RT-PCR for the expression of CD8 and granzymeB in tissue collected from vehicle or GANT61 treated mice. ${ }^{*} P<0.05$ compared to control group, $n=5$ per group. Immunohistochemistry for the expression of CD8+ infiltrating cells in tissue collected from $(\mathbf{F})$ vehicle or $(\mathbf{G})$ GANT61 treated mice. 


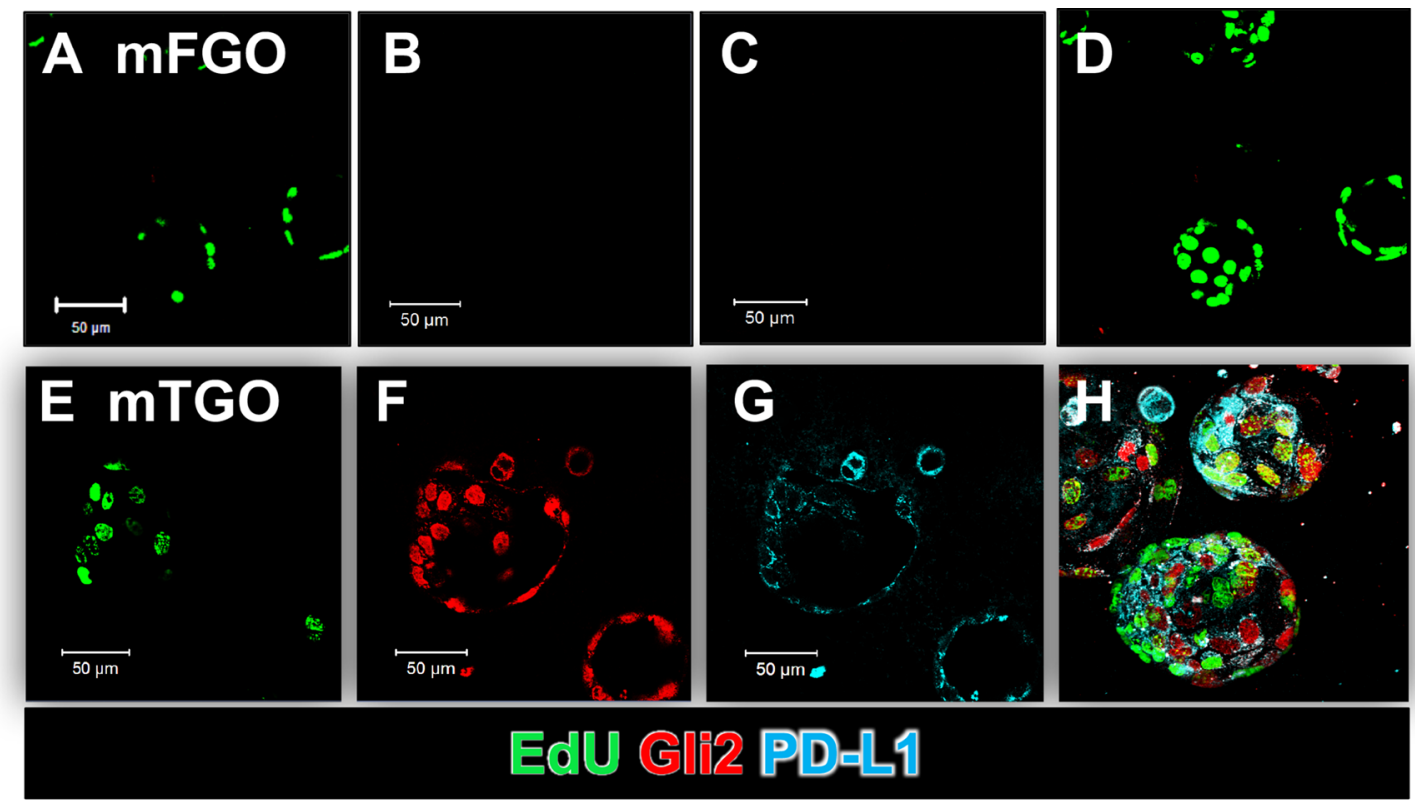

\section{GANT61 $(\mu \mathrm{M})$}
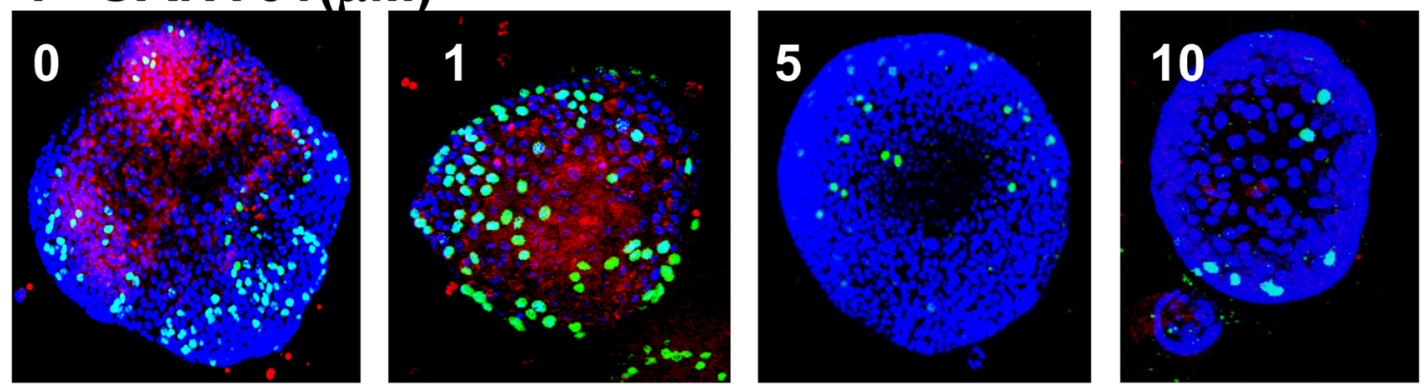

\section{PD=L1 EdU Hळechst}
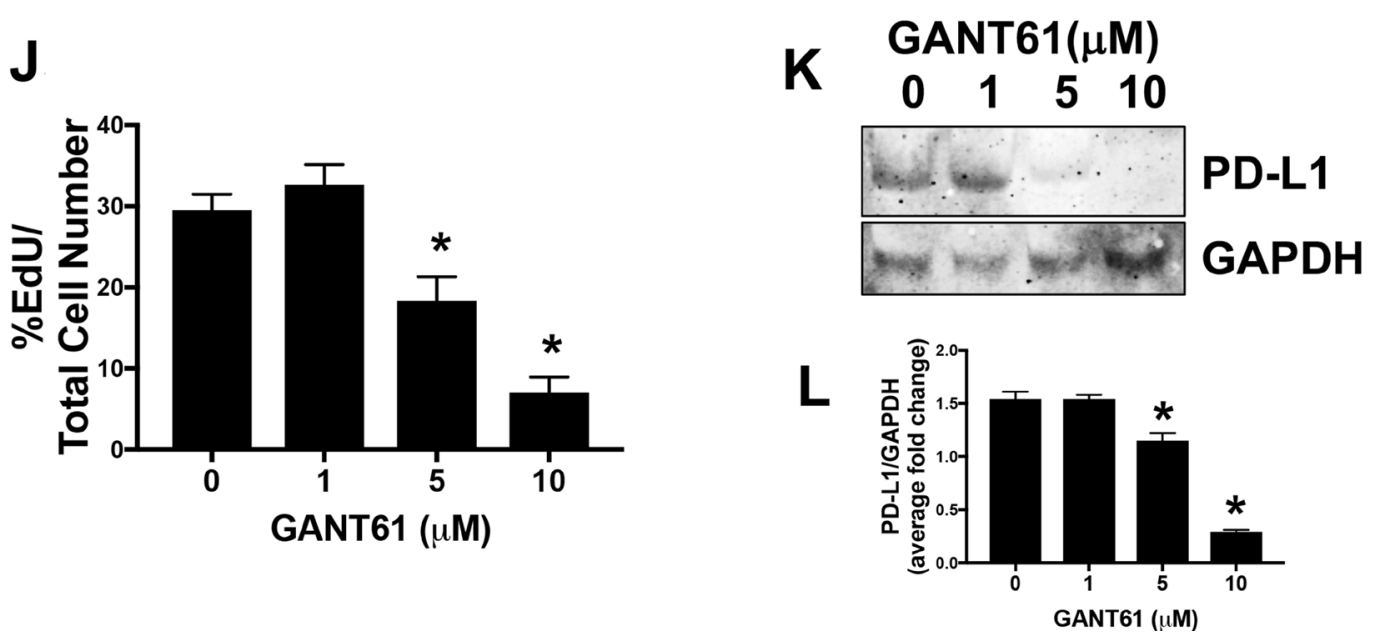

Figure 3: Gli2 and PD-L1 expression in gastric cancer organoids derived from iLgr5;GLI2A mice. Immunofluorescence staining for proliferating EdU (green), Gli2 (red) and PD-L1 (cyan) expression in organoids derived from control normal (mFGO, A-D) and tumor iLgr5; GLI2A-derived (mTGO, E-H) mouse tissues. (I) Immunofluorescence images of PD-L1 (red) and EdU (green) expression in mTGOs, treated with $0,1,5$, or $10 \mu \mathrm{M}$ GANT61. (J) Quantification of \% EdU positive cells/total cells in GANT61 treated mTGOs. ${ }^{*} P<0.05$ compared to $0 \mu \mathrm{M}$ GANT61, $n=4$ per group. (K) Western blot analysis for the expression of PD-L1 using whole cell lysates collected from mTGOs, treated with $0,1,5$, or $10 \mu \mathrm{M}$ GANT61. (L) Quantification of western blots expressed as the PD-L1/GAPDH average fold change relative to $0 \mu \mathrm{M}$ GANT61. ${ }^{*} P<0.05$ compared to $0 \mu \mathrm{M}$ GANT61, $n=4$ per group. 


\section{Secreted tumor antigen induces PD-1 expression on cytotoxic T Cells (CTLs) and protects cancer organoids from CTL-induced apoptosis}

To begin to elucidate the role of Hh-induced PD-L1 expression in cancer cell immune evasion, we next developed an autologous culture of gastric cancer organoids, dendritic cells (DCs) and CTLs. Gastric organoids were generated from normal mouse stomachs (mFGO) or iLgr $5 ;$ GLI2A (mTGO) mice [16]. Conditioned media was collected from cultured $\mathrm{mFGO}^{\mathrm{CM}}$ or $\mathrm{mTGO}^{\mathrm{CM}}$. DCs were pulsed with either $\mathrm{mGO}^{\mathrm{CM}}$ or $\mathrm{mTGO}^{\mathrm{CM}}$ for 24 hours and co-cultured with isolated CTLs for 24 hours. Flow cytometric analysis demonstrated increased expression of PD-1, IFN $\gamma$ and IL-2 in CTLs co-cultured with DCs pulsed with $\mathrm{mTGO}^{\mathrm{CM}}$ (Figure 4A, 4D). However, PD-1, IFN $\gamma$ and IL-2-expressing CTLs co-cultured with DCs pulsed with $\mathrm{mFGO}^{\mathrm{CM}}$ was not significantly different from expression of CTLs co-cultured with unpulsed DC (Figure 4B, 4C). We then co cultured mGOs or mTGOs with CTLS and DCs, either unpulsed or pulsed with $\mathrm{mGO}^{\mathrm{CM}} / \mathrm{mTGO}^{\mathrm{CM}}$ for $48 \mathrm{hrs}$.

Pulsed DCs and TCs were then co-cultured with $\mathrm{mFGOs}$ or mTGOs with and without PD-L1 inhibitor (PDL1I) and apoptosis measured by TUNEL assay (Figure 4E). Organoid/immune cell co-cultures of: 1) mTGOs co-cultured with DCs and CTLs, 2) mTGOs alone with PD-L1I, 3) mTGOs co-cultured with pulsed DCs and CTLs, and 4) mTGOs co-cultured with pulsed DCs and CTLs treated with PDL1I were performed and apoptosis measured (Figure 4F, 4G). Figure 4F demonstrates the migration of immune cells surrounding the mTGOs in coculture in response to pulsed DC and CTLs. Representative time-lapse video is shown in Supplementary Video 1. Interestingly, when co-cultures were pretreated with PDL1 inhibitor there was a significant induction in organoid apoptosis in the wells co-cultured with pulsed DCs (Figure 4F, 4G). Representative time-lapse video is shown in Supplementary Video 2. These data suggest that the inhibition of cancer cell PD-L1 results in cytotoxic T cellinduced tumor apoptosis. Matured DCs were characterized by flow cytometric analysis using specific markers like, CD40, CD80, CD86, CD11c and I-AB (data not shown).

\section{Inhibition of Hh signaling sensitizes human- derived gastric cancer organoids (huTGOs) to chemotherapy in vivo and in vitro}

The human relevance of Hh signaling in gastric cancer cell viability was addressed using a human-derived gastric cancer organoid model previously reported by our group [21]. We generated human-derived gastric cancer organoids (huTGO) from resected tumors obtained from 7 different patients. Figure 5A reports the Gli2 and PD-L1 expression of all lines based on immunofluorescence staining. Based on the immunofluorescence data, we chose 1 organoid line expressing Gli2 and PD-L1 (huTGO2) (Figure 5B, 5C), and a line that did not express either Gli2 or PD-L1 (huTGO7) (Figure 5D, 5E) based on the robustness of the cultures. There was a significant difference in proliferation between these two organoid line (Figure 5F). Gli2 (Figure 5G, 5H) and PD-L1 (Figure 5I, 5J) expression, and proliferation (Figure 5K) within the patient's native tumor tissue was reflected in the organoid cultures.

To assess the role of $\mathrm{Hh}$ signaling in chemoresistance, huTGOs were treated with standardof-care chemotherapeutic drugs epirubicin, oxaliplatin and 5-FU alone or in combination with $\mathrm{Hh} / \mathrm{Gli}$ inhibitor GANT61. Treatment of huTGO2 with GANT61 significantly sensitized the organoids to chemotherapy, as indicated by a shift in the IC50 (Figure 6A, Figure 7A), and induced a significant cell death (Figure 6C). In contrast to huTGO2, treatment of huTGO7 with chemotherapy induced a significant induction in cell death (Figure 6C). GANT61 treatment of huTGO7 did not alter the response to chemotherapy (Figure 6B, 6C, and Figure 7B).

The huTGO2 line was used in a xenograft model. Mice were then treated with either vehicle (PBS), cisplatin (Cis), GANT61 or Cis+GANT61. Histological evaluation revealed the development of adenocarcinoma in mice xenografted with huTGO2 (Figure 8A). Immunofluorescence using an antibody specific for human histone confirmed the engraftment of human-derived cells (Figure 8E). Histology was reflected in tumor volume (Figure 8I). Compared to tumor growth that was observed in vehicle treated mice (Figure 8A, 8E, 8I), Cis (Figure 8B, 8F, 8I) and GANT61 (Figure 8C, 8G, 8I) alone treated mice had significantly decreased tumor volume 21 days post-xenograft. Importantly, combination treatment of Cis+GANT61 was the most effective at inhibiting tumor growth over time (Figure 8D, 8H, 8I). GANT61 (Figure $8 \mathrm{~J}, 8 \mathrm{M}$ ) or Cis+GANT61 combined (Figure $8 \mathrm{~J}, 8 \mathrm{~N}$ ) correlated with decreased tumor cell proliferation of tumor growth. There was no statistically significant difference between the vehicle (Figure 8J, 8K) and Cis alone (Figure $8 \mathrm{~J}, 8 \mathrm{~L}$ ) treated groups.

We investigated the changes in PD-L1 expression in the treated xenograft mouse model transplanted with huTGO2. The development of adenocarcinoma in vehicle treated mice correlated with expression of PD-L1 (Figure $8 \mathrm{E}, 8 \mathrm{O}$ ). While, Cis alone (Figure 8F, 8O) treatment had no effect on PD-L1 expression, GANT61 alone (Figure $8 \mathrm{G}, 8 \mathrm{O}$ ) or in combination with Cis (Figure $8 \mathrm{H}, 8 \mathrm{O}$ ) resulted in decreased PD-L1 expression within tumor tissue collected from the xenografts (Figure 8H, 8O). Decreased PD-L1 expression in response to GANT61 or GANT61+Cis correlated with decreased expression of Hh canonical signaling genes, PTCH1, PTCH2, and GLI1 (Figure 8P). Collectively, these data suggest that in gastric cancer cells expressing Gli2, inhibition of Hh signaling sensitizes resistance to chemotherapy. 


\section{-- pulsed DCs + pulsed DC}
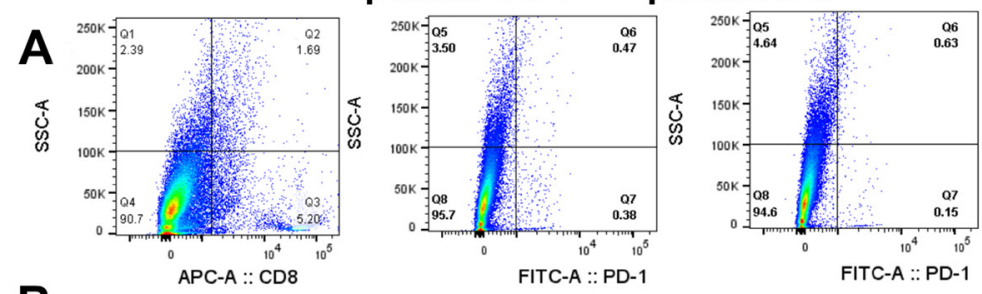

B
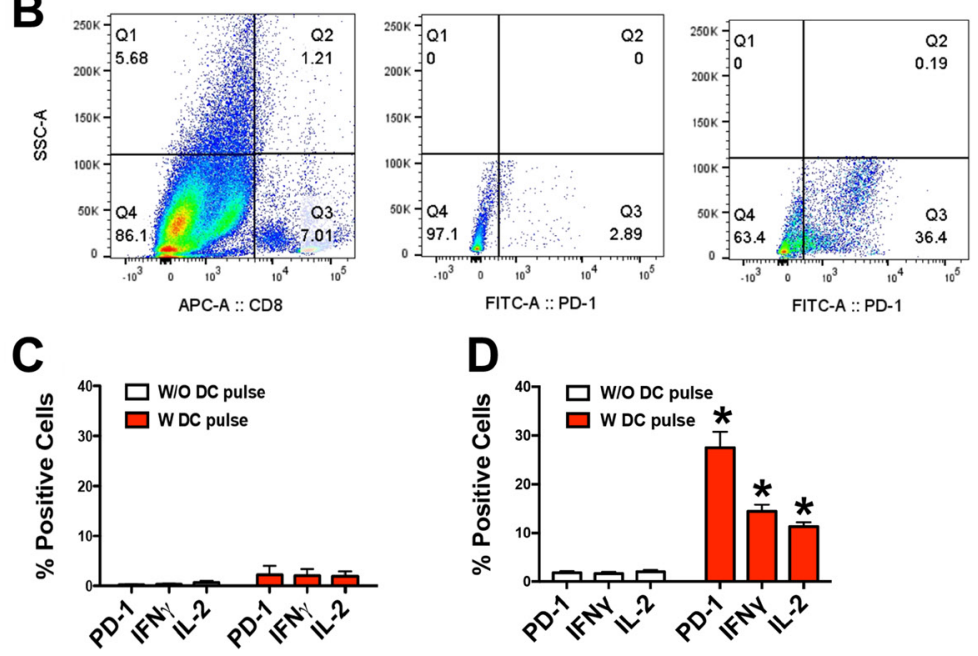

D
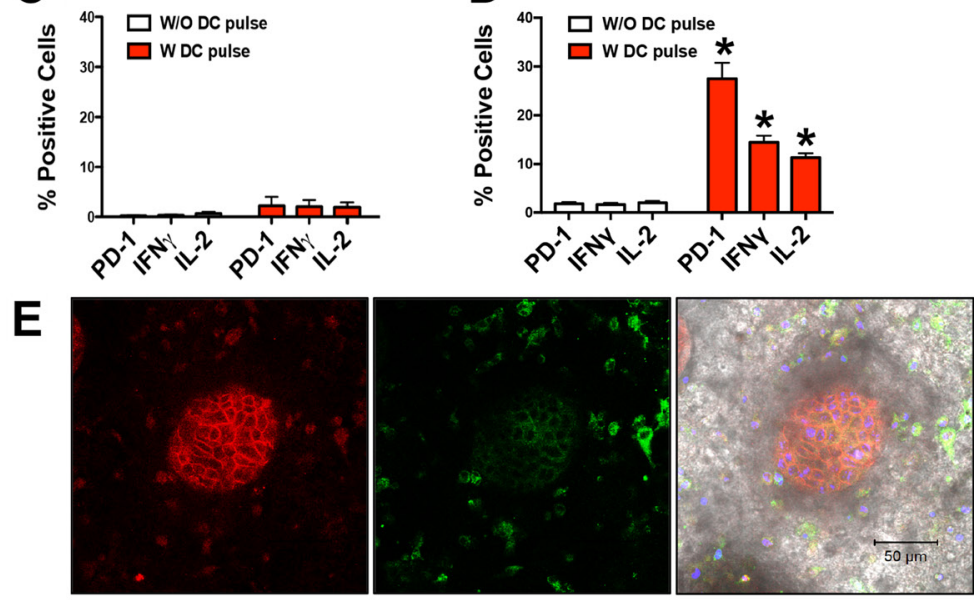

\section{Ecad CD11c Transmitted}

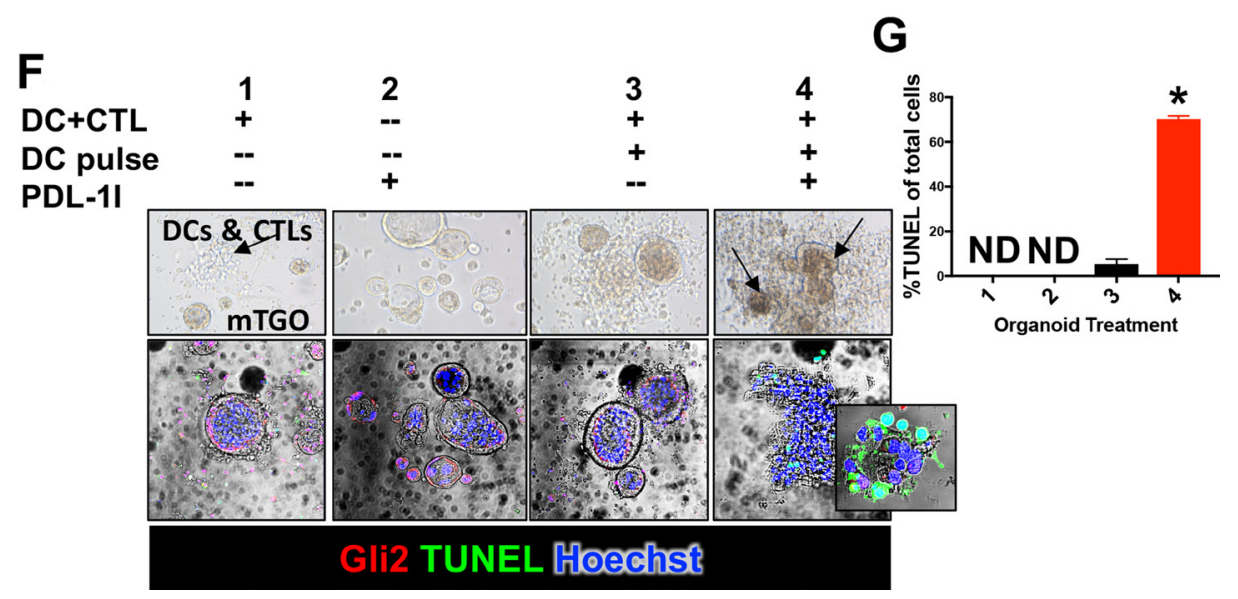

Figure 4: Secreted tumor antigen induces PD-1 expression in cytotoxic T lymphocytes (CTLs) and induce mTGO apoptosis when treated with PD-L1I. Flow cytometric histograms showing the expression of CD8 and PD-1 on CTLs co-cultured with DCs pulsed (+pulsed DC) with either (A) $\mathrm{mGOs}^{\mathrm{CM}}$ or, $(\mathbf{B}) \mathrm{mTGOs}^{\mathrm{CM}}$. The middle and right panels are sub-gated to the $\mathrm{CD} 8+$ cells from the left panels. Quantification of PD-1, IFN $\gamma$ and IL-2-expressing CTLs co-cultured with DCs pulsed with $(\mathbf{C}) \mathrm{mFGOs}^{\mathrm{CM}}$, or (D) $\mathrm{mTGO}^{\mathrm{CM}}$. ${ }^{*} P<0.05$ compared to CTLs exposed to unpulsed DCs, $n=4$ individual organoid/immune cell co-cultures. (E) Representative Immunofluorescence image of $\mathrm{mTGO} / \mathrm{immune}$ cell co-culture stained for the expression of $\mathrm{CD} 11 \mathrm{c}$ (green) and $\mathrm{E}$ cadherin (Ecad, red). (F) TUNEL stain of organoid/immune cell co-cultures of: 1) mTGOs/DCs/CTLs, 2) mTGOs alone with PD-L1I, 3) mTGOs/pulsed DCs and CTLs, and 4) mTGOs/pulsed DCs/CTLs culture treated with PD-L1I. (G) Quantification of apoptotic cells in co-culture conditions 1-4. ${ }^{*} P<0.05$ compared to condition $1, n=4$ individual organoid/immune cell co-cultures. 


\section{DISCUSSION}

Our data show that $\mathrm{Hh}$ signaling plays a role in mediating PD-L1 expression during gastric cancer development. In support of our findings, studies in pancreatic ductal adenocarcinoma cell lines revealed that Hh signaling induced PD-L1 expression under hypoxic conditions [15]. Evidence in the literature also demonstrates that the induction of PD-L1 is largely mediated by proinflammatory cytokines, of which IFN $\gamma$ is the most potent [22]. The intrinsic induction of PD-L1 is mediated via several oncogenic and

\begin{tabular}{|l|c|c|}
\hline \multicolumn{2}{|c|}{ A } & Expression of Gli2 and PD-L1 in TGO lines generated \\
\hline Organoid Line & Gli2 +ve & PD-L1 +ve \\
\hline TG01 & + & + \\
\hline TG02 & + & + \\
\hline TG03 & Line lost before analysis & \\
\hline TG04 & + & + \\
\hline TG05 & + & + \\
\hline TG06 & - & - \\
\hline TG07 & - & - \\
\hline
\end{tabular}
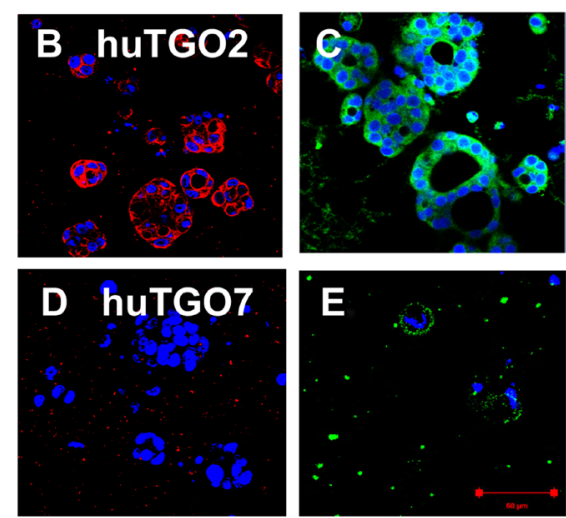

PD=ட1 티=2 Hهechst
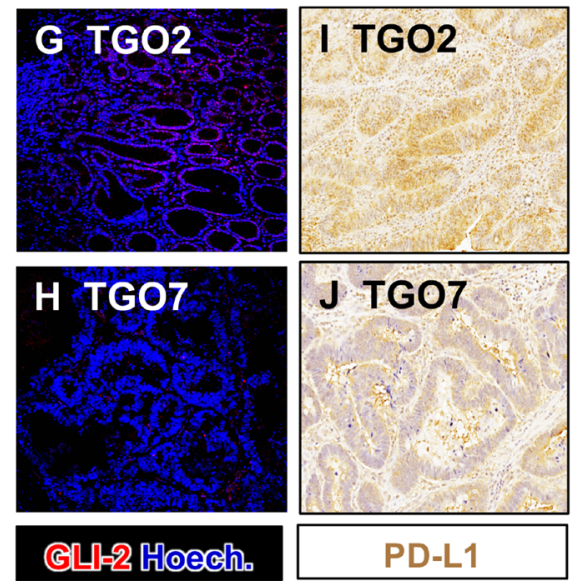

$\mathbf{F}$

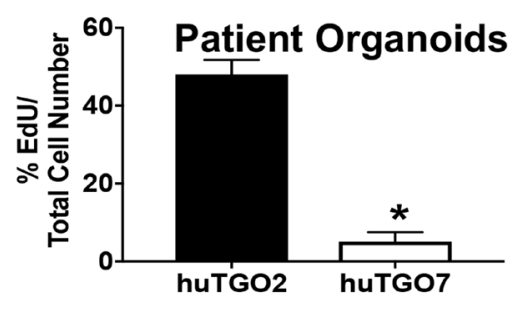

K

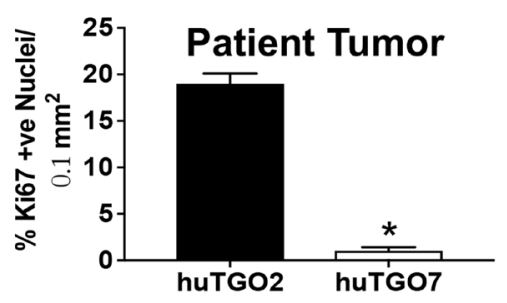

Figure 5: Expression of Gli2 and PD-L1 in huTGO lines and patient tumor tissue. (A) Expression of Gli2 and PD-L1 in TGO lines generated. Immunofluorescence images for the expression of PD-L1 (green) and Gli2 (red) in (B, C) huTGO2, and (D, E) huTGO7. (F) Quantification of proliferating cells within huTGO2 and huTGO7 organoid lines. ${ }^{*} P<0.05$ compared to huTGO2, $n=4$ individual organoids. Immunofluorescence staining for Gli2 (red) in patient tumor tissue from which (G) huTGO2 and (H) huTGO7 were derived. Immunohistochemistry for the expression of PD-L1 in patient tumor tissue from which (I) huTGO2 and (J) huTGO7 were derived. (K) Proliferation in patient tumor tissue from which huTGO2 and huTGO7 were derived. ${ }^{*} P<0.05$ compared to tumor tissue from patient from which huTGO2 was derived, $n=6$ individual high power fields. 
transcriptional pathways. For example, in glioblastoma PD-L1 upregulation correlates with loss of PTEN [23]. In addition, activation of PI3K and mTOR pathway, or the epidermal growth factor receptor/mitogen-activated protein kinase pathway correlates with the overexpression of PD-L1 [24, 25]. In a co-culture of Panc-1 cells with lymphocytes, blockade of PD-L1, with either anti-PD-L1 antibody or $\mathrm{Hh}$ inhibitor cyclopamine, resulted in induced lymphocyte anti-tumor activity [15]. We extend these findings both in vivo using a mouse model of gastric cancer, and in vitro using a gastric cancer organoid culture. In vivo, in a mouse model of gastric cancer that was developed by overexpressing GLI2A in Lgr5+ gastric stem cells (iLgr5;GLI2A mice) [16], GANT61 inhibition of adenocarcinoma correlated with two effects that included reduced 1) PD-L1 expression, and 2) tumor proliferation that resulted in increased CD8+ cytotoxic T lymphocytes. These data suggest that GANT61 partially mediates tumor suppression via reduced PD-L1 expression. This is evident from the findings in Figures 6 and 8, whereby in Figure 6 there is an absence of CTL and thus we may consider that organoid death is due to loss of proliferation. In contrast, in Figure 8 in vivo the effect of GANT61 on tumor growth may also be mediated by inhibition of PD-L1. Moroever, in vitro using gastric cancer organoids derived from iLgr5; GLI2A mice, GANT61 similarly reduced PD-L1 expression and cancer cell proliferation. Interestingly, in vivo GANT61 inhibition of Gli2-induced PD-L1 by tumor cells, resulted in increased CTL numbers within the tumor tissue. The PD-1/PD-L1 pathway is involved

\section{A huTGO2}
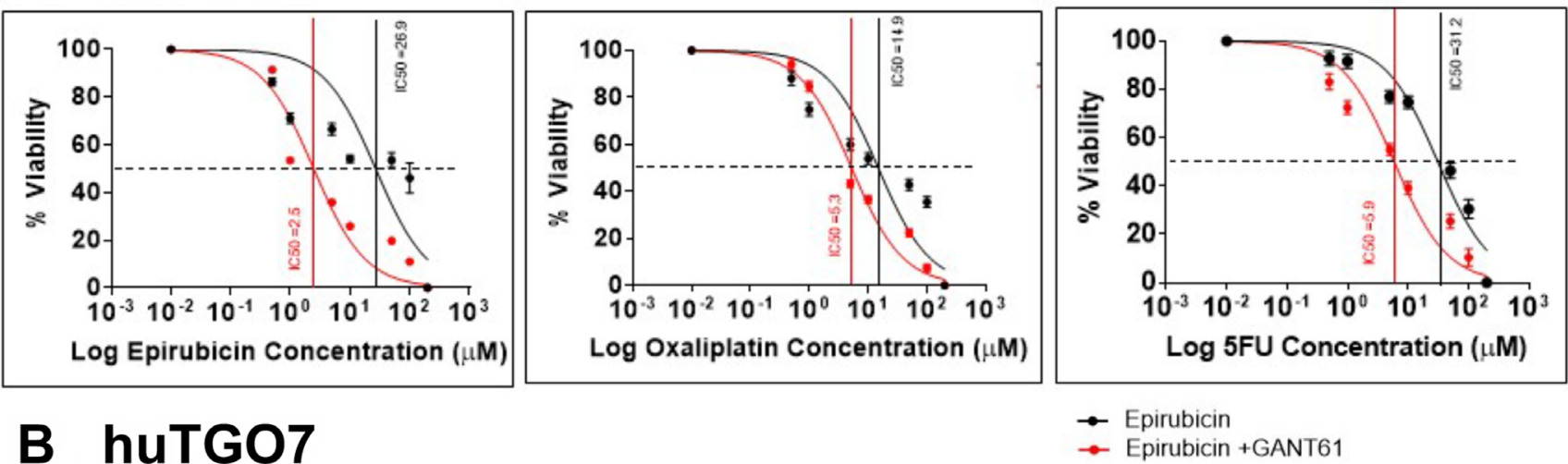

\section{B huTGO7}
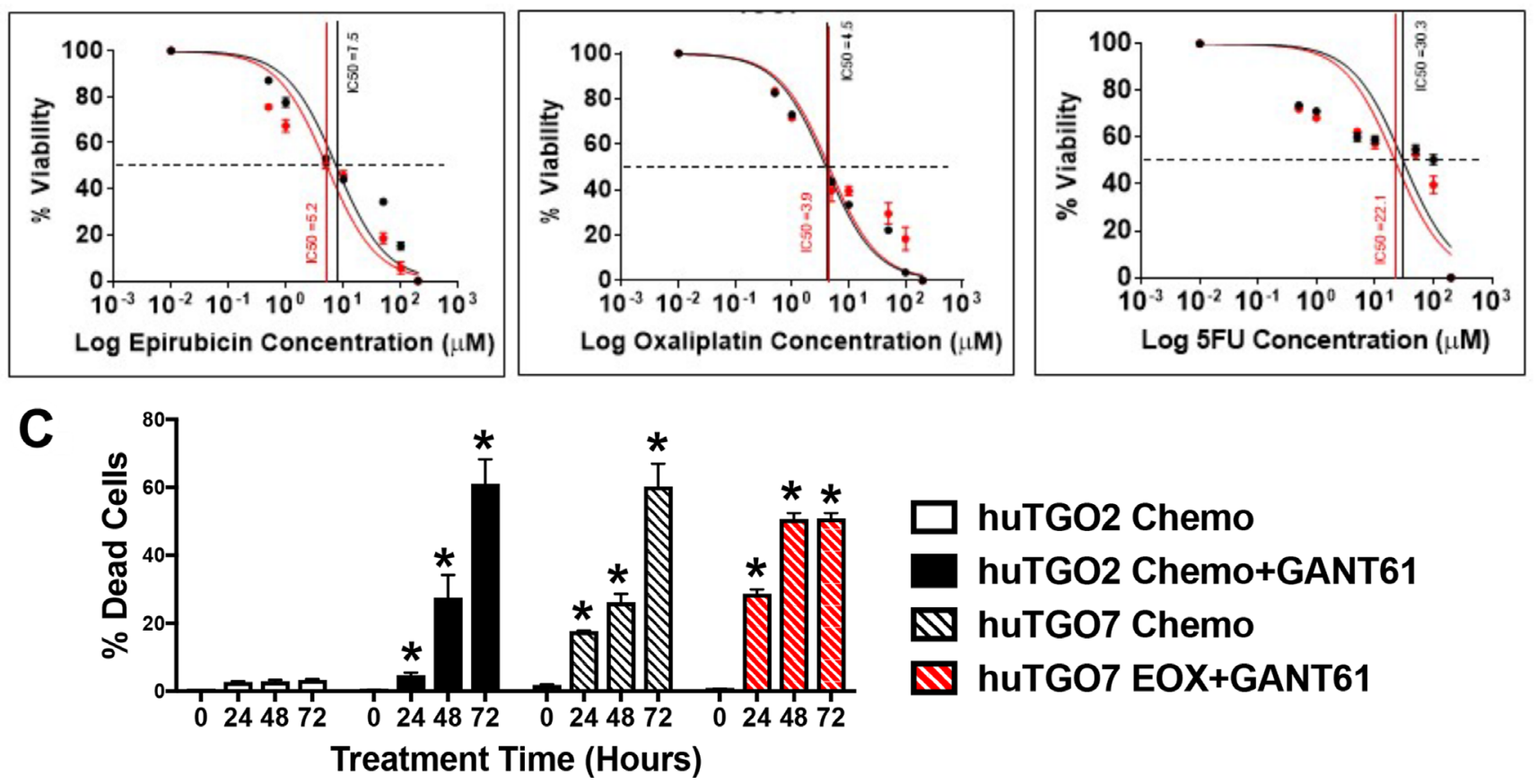

Figure 6: Response of human-derived gastric cancer organoids (huTGO2 and huTGO7) to standard-of-care chemotherapeutic drugs and Hh/Gli inhibitor GANT61. Dose responses epirubicin, oxaliplatin, and 5-Flurouracil (5FU) alone (black curve) or in combination with Hh inhibitor GANT61 (red curve) using (A) huTGO2 or (B) huTGO7 organoid line. (C) Quantification of organoid cell death in response to combination chemotherapy (epirubicin, oxaliplatin and 5FU) alone or with GANT61 using huTGO2 or huTGO7 lines over a 72 hour treatment. ${ }^{*} P<0.05$ compared to 0 hour treatment, $n=3$ individual organoid assays. 
A huTGO2

\begin{tabular}{|l|l|l|}
\hline $\log$ (inhibitor) vs. normalized response & \multicolumn{1}{|c|}{ Epirubicin } & Epirubicin +GANT61 \\
\hline LogIC50 & $1.43 \pm 0.12$ & $0.3917 \pm 0.07$ \\
\hline IC50 & $26.9 \pm 0.12$ & $2.5 \pm 0.07$ \\
\hline $95 \% \mathrm{Cl}$ (profile likelihood) & & \\
\hline LogIC50 & 1.07 to 1.74 & 0.2432 to 0.541 \\
\hline IC50 & 11.75 to 54.99 & 1.751 to 3.476 \\
\hline
\end{tabular}

\begin{tabular}{|l|l|l|}
\hline $\log ($ inhibitor) vs. normalized response & \multicolumn{1}{|c|}{ Oxaliplatin } & Oxaliplatin +GANT61 \\
\hline Log|C50 & $1.2 \pm 0.09$ & $0.7204 \pm 0.04$ \\
\hline IC50 & $14.9 \pm 0.09$ & $5.253 \pm 0.04$ \\
\hline $95 \% \mathrm{Cl}$ (profile likelihood) & & \\
\hline Log IC50 & 0.914 to 1.439 & 0.6383 to 0.8024 \\
\hline IC50 & 8.203 to 27.47 & 4.348 to 6.345 \\
\hline
\end{tabular}

\begin{tabular}{|l|l|l|}
\hline log(inhibitor) vs. normalized response & \multicolumn{1}{|c|}{ 5FU } & \multicolumn{1}{|c|}{ 5FU +GANT61 } \\
\hline LogIC50 & $1.493 \pm 0.05$ & $0.7722 \pm 0.05$ \\
\hline IC50 & $31.15 \pm 0.05$ & $5.918 \pm 0.05$ \\
\hline $95 \% \mathrm{Cl}$ (profile likelihood) & & \\
\hline LogIC50 & 1.383 to 1.6 & 0.6438 to 0.8989 \\
\hline IC50 & 24.16 to 39.84 & 4.404 to 7.924 \\
\hline
\end{tabular}

\section{B huTG07}

\begin{tabular}{|l|l|l|}
\hline $\log$ (inhibitor) vs. normalized response & \multicolumn{1}{|c|}{ Epirubicin } & \multicolumn{1}{|c|}{ Epirubicin +GANT61 } \\
\hline Log|C50 & $0.8768 \pm 0.06$ & $0.7133 \pm 0.06$ \\
\hline IC50 & $7.53 \pm 0.06$ & $5.168 \pm 0.06$ \\
\hline $95 \% \mathrm{Cl}$ (profile likelihood) & & \\
\hline LogIC50 & 0.7246 to 1.03 & 0.5526 to 0.8682 \\
\hline IC50 & 5.304 to 10.73 & 3.57 to 7.382 \\
\hline
\end{tabular}

\begin{tabular}{|l|l|l|}
\hline $\log$ (inhibitor) vs. normalized response & \multicolumn{1}{|c|}{ Oxaliplatin } & Oxaliplatin +GANT61 \\
\hline LogIC50 & $0.6012 \pm 0.04$ & $0.6512 \pm 0.07$ \\
\hline IC50 & $3.992 \pm 0.04$ & $4.48 \pm 0.07$ \\
\hline $95 \% \mathrm{Cl}$ (profile likelihood) & & \\
\hline LogIC50 & 0.5012 to 0.6999 & 0.4716 to 0.8293 \\
\hline IC50 & 3.171 to 5.01 & 2.962 to 6.75 \\
\hline
\end{tabular}

\begin{tabular}{|l|l|l|}
\hline $\log$ (inhibitor) vs. normalized response & \multicolumn{1}{|c|}{$5 \mathrm{FU}$} & \multicolumn{1}{|c|}{ 5FU +GANT61 } \\
\hline LogIC50 & $1.482 \pm 0.14$ & $1.344 \pm 0.13$ \\
\hline IC50 & $30.33 \pm 0.14$ & $22.06 \pm 0.13$ \\
\hline $95 \% \mathrm{Cl}$ (profile likelihood) & & \\
\hline LogIC50 & 1.044 to 1.83 & 0.9507 to 1.691 \\
\hline IC50 & 11.06 to 67.55 & 8.928 to 49.06 \\
\hline
\end{tabular}

Figure 7: Reported IC50 and 95\% CI for doses in response to chemotherapy treatment with GANT61. IC50 and 95\% CI for in response to chemotherapeutic drugs alone or in combination with GANT61 using (A) huTGO2 or (B) huTGO7 lines. 

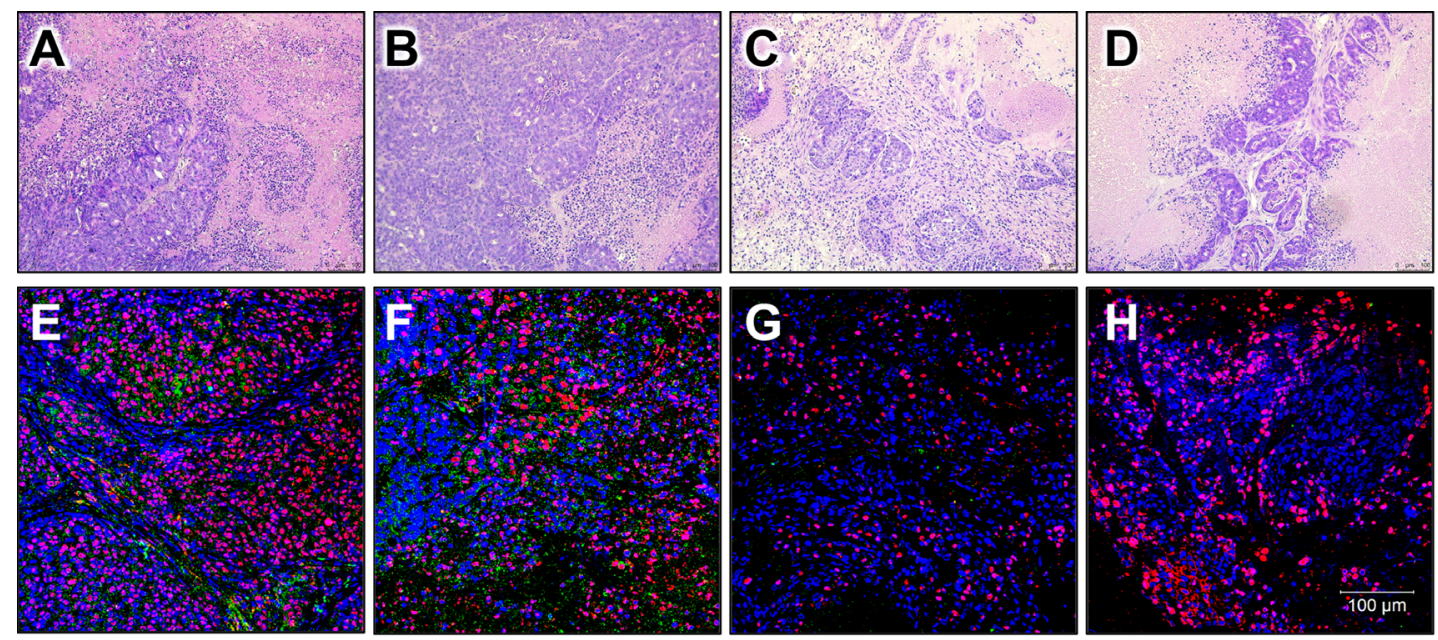

PD-L1 huHistone Høeahst
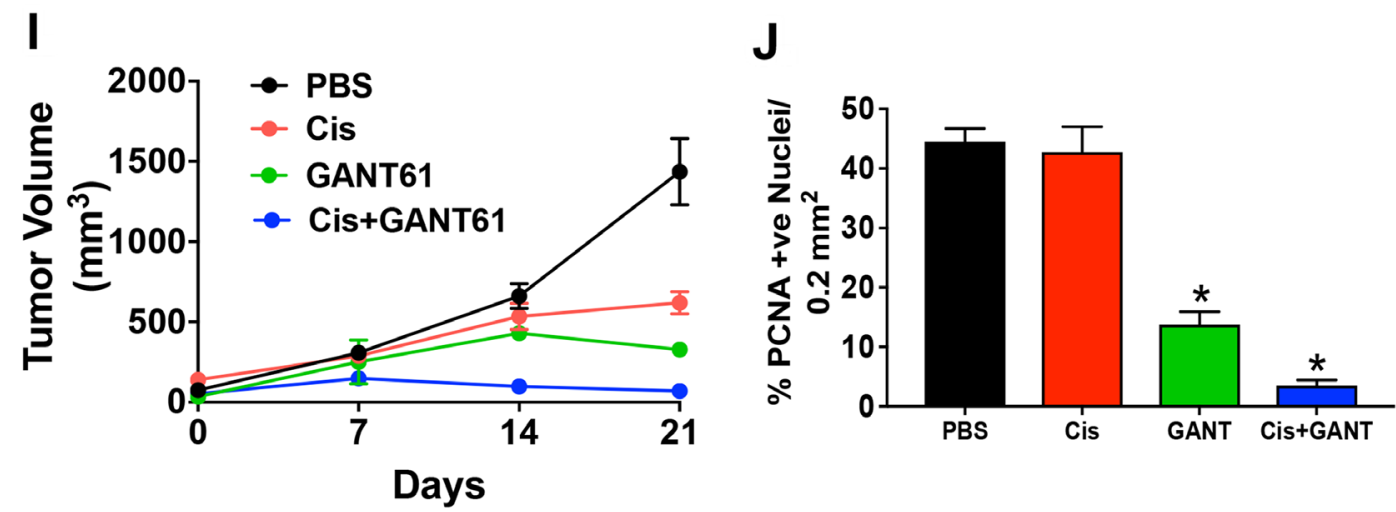

\section{K PBS}

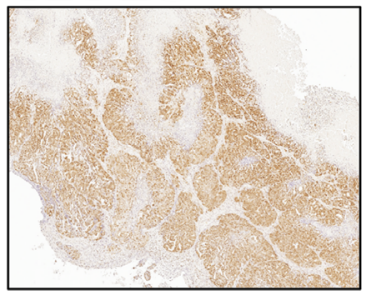

0

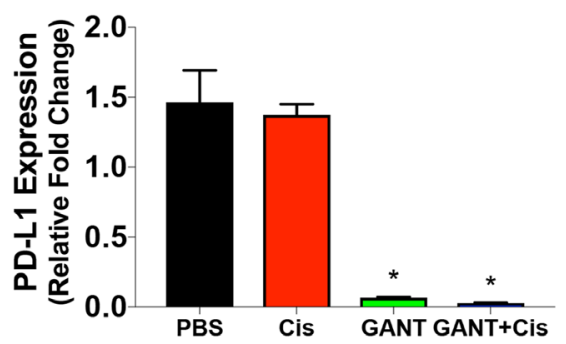

L Cis

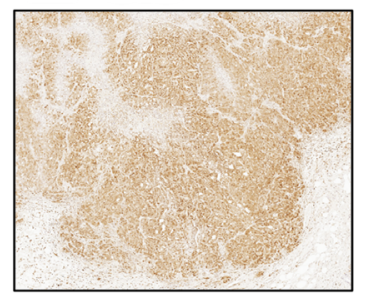

M GANT

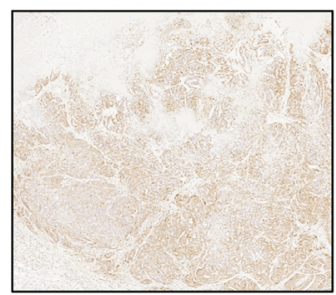

\section{N Cis+GANT}

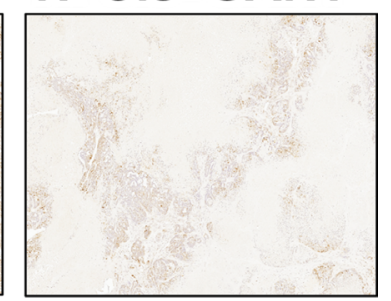

$\mathbf{P}$

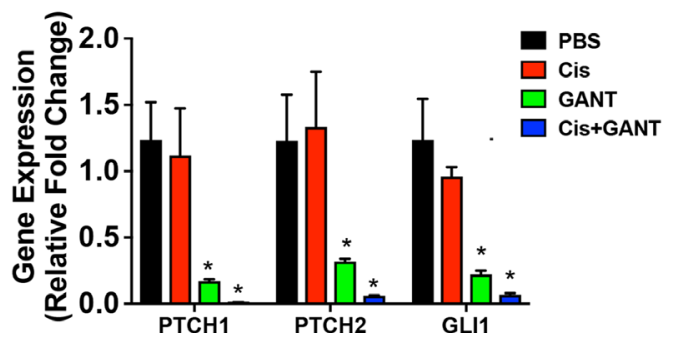

Figure 8: Xenograft mouse model developed using human-derived gastric cancer organoids (huTGO2). H\&E and immunofluorescence staining for PD-11 (green and human-specific histone (huHistone, red) of sections collected from xenografted mice treated with either (A, E) vehicle, (B, F) Cisplatin (Cis), (C, G) GANT61 or (D, H) Cis + GANT61. (I) Tumor volume $\left(\mathrm{mm}^{3}\right)$ measured in huTGO2 xenograft model. (J) Percentage of PCNA positive nuclei per $0.1 \mathrm{~mm}^{2}$ section. Representative immunohistochemical stains of sections collected from xenografted mice treated with either $(\mathbf{K})$ vehicle, (L) Cis, (M) GANT61 or (N) Cis+ GANT61. Quantitative RT PCR measuring the expression of (O) PD-L1 and (P) canonical Hh signaling genes, PTCH1, PTCH2 and GLI1. ${ }^{*} P<0.05$ compared to vehicle/PBS group, $n=5$ per group. 
in controlling the proliferation and cytokine production of T cells [26]. Thus, a plausible explanation may be that the observation is attributed to increased proliferation of resident CTLs within the tumor tissue rather than infiltration of $\mathrm{CD} 8+$ cells. Overall, the relationship between PD-L1 expression and prognosis in gastric cancer is still controversial [27]. While some studies show that PD-L1 expression correlates with an adverse prognosis, others show that patients with PD-L1 positive tumor cells have an improved prognosis [28, 29]. Therefore, such information makes it difficult to predict the effectiveness of immune-checkpoint inhibition for the treatment of gastric cancer.
Here, we generated an autologous organoid/immune cell co-culture to understand the feasibility for the use of such a model to potentially predict the efficacy of immune-checkpoint inhibition for the treatment of gastric cancer. The first significant observation that we made from this culture was that secreted tumor antigen induced PD-1 expression on CTLs. When antigen presenting dendritic cells were pulsed with conditioned media collected from gastric cancer mTGOs and co-cultured with CTLs, we observed a significant induction of PD-1 on these lymphocytes. Second, the co-culture of CTLs exposed to tumor-specific antigen with mTGOs resulted in the protection of cancer cells from anti-tumor lymphocyte

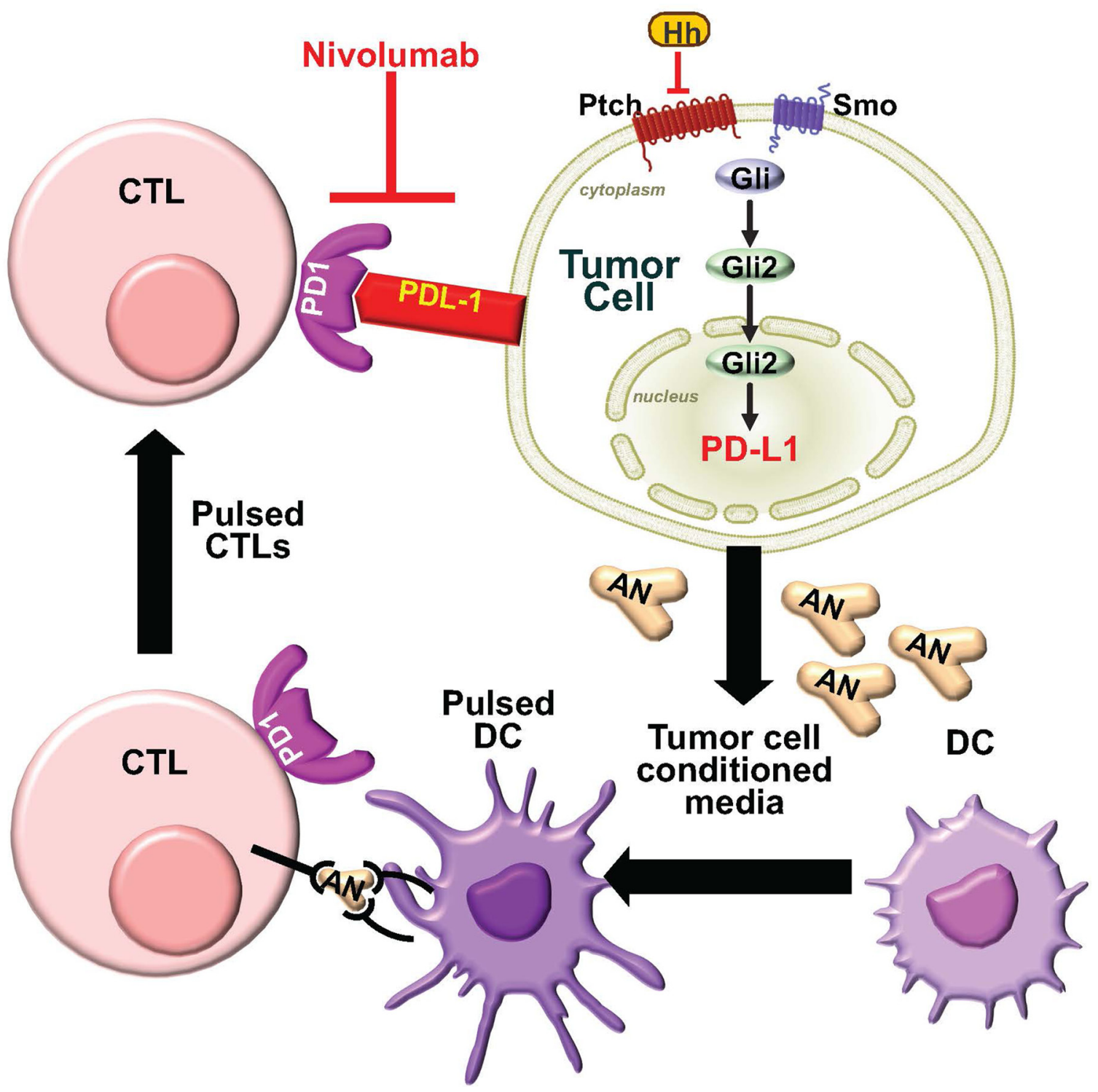

Figure 9: Proposed model for the induction of PD-L1 by Hh signaling. We proposed from these studies that Hh signaling induces the expression of PD-L1 on gastric tumor cells. When antigen presenting dendritic cells (DC) are pulsed with conditioned media collected from gastric cancer organoids, containing tumor-specific antigen (AN), and co-cultured with CTLs, there is a significant induction of PD-1 on these lymphocytes. Thus, secreted tumor-specific AN induces PD-1 expression on CTLs. The co-culture of CTLs exposed to tumor-specific AN with gastric cancer organoids resulted in the protection of cancer cells from anti-tumor lymphocyte activity. Immunecheckpoint inhibition using anti-PD-1 inhibitor Nivolumab resulted in cancer organoid death. 
activity. Immune-checkpoint inhibition resulted in mTGO death. Tumor-associated antigens are antigens expressed on tumor cells that can elicit an immune response within the tumor microenvironment. In order to induce tumorspecific $\mathrm{T}$ cells, immunogenic peptides derived from tumor-associated antigens must be presented to $\mathrm{T}$ cells by efficient professional antigen-presenting cells, such as dendritic cells, which are able to activate naïve and memory $\mathrm{T}$ cells $[30,31]$. Under ideal circumstances, immunogenic peptides derived from tumor-associated antigens presented on the surface of tumor cells with MHC-I activate cytotoxic $\mathrm{T}$ cells and subsequently results in the lysis of tumor cells [31, 32]. As our data suggests, the tumor-associated antigen secreted within the conditioned media of mTGOs induced PD-1 on CTLs leading to immune evasion by the cancer cells. The overall goal of the study was to develop a culture system to test whether inhibition of Gli2-PDL1 would lead to tumor cell apoptosis. To test this hypothesis, the development of an autologous model was necessary, in which tumor antigens were used to cross-present to CTLs. The result of these studies showed that PD-1/PD-L1 inhibition reversed the tolerance induced by Gli2. The mouse organoid/ immune cell co-culture may be a promising preclinical model. Future studies using patient-derived gastric cancer organoids and autologous patient-derived immune cells in such a co-culture may be beneficial to testing immunotherapy for each individual patient. Importantly, we report that Hh signaling contributes to the induction of PD-L1 expression in gastric cancer, suggesting that combinatorial drug therapy using $\mathrm{Hh}$ signaling and immune checkpoint inhibition may be suitable for candidate patients (Figure 9).

To investigate the role of Hedgehog signaling in the regulation of PD-L1 expression, we used in vivo and in vitro organoid cultures derived from gastric cancer patients. Human-derived gastric cancer organoids (huTGOs) that expressed Gli2, not only highly expressed PD-L1, but were also chemoresistant to standard-of-care drugs. Combinatorial drug treatment of huTGOs with both Hh inhibitor GANT61 and chemotherapeutic drugs resulted in decreased cancer cell proliferation and induced cell death both in vitro and in vivo. These data suggest that activation of the $\mathrm{Hh}$ signaling pathway renders cancer cells resistant to immunotherapy. In support of our findings, Activation of $\mathrm{Hh}$ pathway components including Smo, PTCH and Glil in ovarian epithelial cells resulted in cisplatin resistance [33]. In a separate experiment, coadministration of IPI-926, a drug that depletes tumorassociated stromal tissue by inhibiting the Hh signaling pathway in a xenograft model of pancreatic ductal adenocarcinoma enhanced the delivery of gemcitabine, decreased tumor cell proliferation and disease progression [34]. Treating the patient's organoids together with the patient from which the cultures were derived will potentially test the advantage of this approach to predict the best therapeutic option. Overall, our data presents new avenues for improving the efficacy of therapeutics in patients with gastric cancer.

\section{MATERIALS AND METHODS}

\section{Mice}

All mouse studies were approved by the University of Cincinnati Institutional Animal Care and Use Committee (IACUC) that maintains an American Association of Assessment and Accreditation of Laboratory Animal Care (AAALAC) facility. We used a triple-transgenic model engineered to express an activated GLI2 allele, GLI2A, in Lgr5- expressing stem cells and their progeny in adult mice when treated with tamoxifen followed by doxycycline [16]. The triple-transgenic Lgr5EGFP-IRES-CreERT2; R26-LSL-rtTA-IRES-EGFP; tetOGLI2A mouse is abbreviated as $i \operatorname{Lgr} 5 ;$ GLI2A. ILgr5; GLI2A mice were treated with either vehicle (PBS, i.p., every alternate day), or GANT61 (50 mg/kg body weight, i.p., every alternate day; Stem Cell Technologies, 73692), after the completion of oral doxycycline treatment for 3 weeks. Stomach tissues were collected and analyzed for histology, immunostaining and qRT PCR.

\section{Generation of mouse and human organoids}

Mouse-derived gastric cancer organoids (iLgr5; $G L I 2 A$ mTGOs) were generated from the stomachs using a previously published protocol [21]. Briefly, tumor tissue was collected in cold Dulbecco's phosphate buffered saline (DPBS) without calcium or magnesium (w/o Ca/ $\mathrm{Mg}$ ) and with $1 \%$ penicillin/streptomycin, $1 \%$ kanamycin and Amphotericin B $(0.25 \mathrm{mg} / \mathrm{ml}) /$ Gentamicin $(10 \mathrm{mg} /$ $\mathrm{ml})$. The tissues were minced and incubated in $10 \mathrm{~mL}$ prewarmed EDTA stripping buffer (HBSS with 20\% FCS, $1 \%$ pen/step, HEPES and EDTA) at $37^{\circ} \mathrm{C}$ in a rotating shaker, for $10 \mathrm{~min}$ followed by another $5 \mathrm{~min}$ with fresh EDTA buffer. Tissue was washed with Dulbecco's Modified Eagle's medium (DMEM) supplementated with $1 \%$ penicillin/ streptomycin, $1 \%$ kanamycin and Amphotericin B $(0.25 \mathrm{mg} / \mathrm{ml}) /$ Gentamicin $(10 \mathrm{mg} / \mathrm{ml})$ twice and incubated with $10 \mathrm{~mL}$ pre-warmed collagenase A-hyaluronic acid buffer at $37^{\circ} \mathrm{C}$ in a rotating shaker for 15-30 min. Digested tissue was then filtered through 40 $\mu \mathrm{m}$ filter. Filtered cells were centrifuged at $1200 \mathrm{rpm}$ for $5 \mathrm{~min}$, washed with DPBS and centrifuged at 400xg for $5 \mathrm{~min}$. The supernatant was discarded and the pellet was resuspended in the desired amount of Matrigel ${ }^{\mathrm{TM}}$ supplemented with 1\% Kanamycin and 1\% Penicillin/ streptomycin and seeded into culture plates overlaid with gastric organoid media ((DMEM/F12 supplemented with $10 \mathrm{mM}$ HEPES, 1X Glutamax, 1\% Pen/Strep, 1X N2, 1X B27, 1 mM N-Acetylcystine, $50 \mathrm{ng} / \mathrm{mL}$ EGF, 100 ng/mL Noggin, 10\% R-Spondin Conditioned Media, 
50\% Wnt Conditioned Media, 100 ng/mL FGF10, 10 $\mathrm{nM}$ Gastrin, $10 \mathrm{uM} \mathrm{Y-27632)} \mathrm{and} \mathrm{incubated} \mathrm{at} 37^{\circ} \mathrm{C}$ $\mathrm{CO}_{2}$ incubator. Normal mouse gastric organoids (mGO) were generated from mouse stomach using a previously published protocol [35]. Human tumor tissue was obtained from patients undergoing surgical resection for gastric cancer after consent (IRB protocol number: 20155537, University of Cincinnati). Tumor organoids were generated as previously published protocol [21].

\section{Generation of bone marrow-derived dendritic cells}

Bone marrow cells were collected from either control or $i \operatorname{Lgr} 5 ; G L I 2 A$ mice. The femur and tibia were collected and separated without disturbing the bone marrow. The bone marrow was flushed into a $50 \mathrm{ml}$ tube using a 26G needle and R10 media (RPMI-1640, Fisher Scientific, 10041CV supplemented with, 1\% Penicillin/ streptomycin, and, 10\% FCS), filtered through $40 \mu \mathrm{m}$ filter, centrifuged at $423 \mathrm{~g}$ for 5 minutes at $4{ }^{\circ} \mathrm{C}$. Cells were resuspended in complete dendritic cell (DC) media (RPMI-1640 supplemented with $50 \mu \mathrm{M} \beta$-mercaptoethanol (ThermoFisher Scientific, \#21985023), 1\% Penicillin/ streptomycin, 10\% FCS and, $20 \mathrm{ng} / \mathrm{mL} \mathrm{rmGMCSF} \mathrm{(R \& D}$ System, 415-mL-010)) and plated $1 \mathrm{~mL}$ of cells/well of a 6 well tissue culture plate. Fresh DC media was added to each well on day 3 of the culture [36]. DCs were cultured for 5 days before pulsed with organoid conditioned media (CM).

\section{Isolation and cryopreservation of mouse splenocytes and culture of cytotoxic $T$ lymphocytes (CTLs)}

Spleens were collected from either control or iLgr 5; GLI2A mice in cold PBS/2\% FBS. Each spleen was placed on top of $40 \mu \mathrm{m}$ cell strainer and disrupted with a flat plunger tip of a $10 \mathrm{~mL}$ syringe. Splenocytes were centrifuged at $300 \mathrm{xg}$ for 10 minutes and resuspend in freezing medium $\left(1 \times 10^{8}\right.$ cells/mL RPMI supplemented with $65 \% \mathrm{FCS}$ and $10 \% \mathrm{DMSO}$ ), and frozen at $-80^{\circ} \mathrm{C}$ until organoids were ready for co-culture. Frozen splenocytes were quickly thawed in a $37^{\circ} \mathrm{C}$ water bath in pre-warmed thawing media (RPMI supplemented with $10 \% \mathrm{FCS}$, and, $1 \%$ Penicillin/streptomycin). The cells were centrifuge at $270 \mathrm{xg}$ for $5 \mathrm{~min}$, CTLs were then isolated using the EasySepTM Mouse CD8+ T cell Enrichment according to manufacturer's protocol (Stemcell Technologies \# 19853). CD8+ T cells (CTLs) were resuspended in T-Cell media (RPMI-1640 supplemented with $50 \mu \mathrm{M} \beta$-mercaptoethanol (Thermo Fisher Scientific, \#21985023), 1\% ITS (1.7 $\mu \mathrm{M}$ Insulin, 68.8 $\mu \mathrm{M}$ Transferrin, 3.9 nM Selenite; ThermoFisher Scientific, \#41400045), 1\% Penicillin/ streptomycin, 10\% FCS, $0.25 \mathrm{mg} / \mathrm{ml}$ Amphotericin B /10 $\mathrm{mg} / \mathrm{mL}$ Gentamicin, $50 \mathrm{mg} / \mathrm{ml}$ Kanamycin, 30U/mL (6 $\mathrm{ng} / \mathrm{mL}$ ) mouse recombinant interleukin 2 (IL-2, Mitenyl Biotec, 130-094-055) and, $5 \mathrm{ng} / \mathrm{mL}$ mouse recombinant interleukin 7 (IL-7, Stemcell Technologies, 78054-1)) and cultured in a 6 well plate at a density of $2 \times 10^{6}$ cells/well for 24 hours before co-culture.

\section{Organoid/immune cell co-culture}

After 5 days of culture, DCs were harvested, resuspended in $750 \mu \mathrm{L}$ DC media and seeded back to same wells with $100 \mu \mathrm{L}$ of conditioned media collected from normal/control (mGO ${ }^{\mathrm{CM}}$ ) or iLgr5;GLI2A mouse-derived $\left(\mathrm{mTGO}^{\mathrm{CM}}\right)$ organoids. DCs were pulsed with conditioned media for 24 hours. On the same day, CTL culture was started. After 24 hours of culture, CTLs were centrifuged at $270 \mathrm{xg}$ for $5 \mathrm{~min}$ and resuspend in $750 \mu \mathrm{L}$ TC media and added to the matured and pulsed DCs. At this stage IL-7 was withdrawn from the wells where conditioned media was added. Pulsed DCs and CTLs were co-cultured for 24 hours.

Suspended or loosely adherent DCs and CTLs were harvested by centrifugation. Adherent DCs were harvested using cell dissociation buffer $(1 \mathrm{~mL} /$ well, ThermoFisher Scientific, 13151-014). At that time mGOs or mTGOs were harvested in cold PBS, centrifuged at $406 \mathrm{xg}$ for 5 min and resuspended in cold PBS combined with DCs and CTLs. Organoids, DCs and CTLs were centrifuged at $406 \mathrm{xg}$ for $5 \mathrm{~min}$ and resuspended in the desired amount of Matrigel ${ }^{\mathrm{TM}}$. A $30 \mu \mathrm{L}$ Matrigel $^{\mathrm{TM}}$ bubble was seeded in each well overlaid with gastric organoid media. Experimental groups included: 1) mGOs or mTGOs plus DCs and CTLs, 2) mGOs or mTGOs alone treated with PD-1 inhibitor (270 pg/mL, Nivolumab, Selleckchem, A2002), 3) mGs or mTGOs plus pulsed DCs and CTLs, and 4) mGs or mTGOs plus pulsed DCs and CTLs treated with PD-L1 inhibitor in 8 well chambered coverglass. Organoids were analyzed by TUNEL staining according to the manufacturer's protocol (Thermo Fisher scientific, C10245). A time-lapse video with brightfield images were acquired every 15 min up to $16 \mathrm{hrs}$ using Inverted Zeiss LSM 710 confocal microscope, equipped with $37^{\circ} \mathrm{C}$ incubator and $5 \% \mathrm{CO}_{2}$.

\section{Flow cytometric analysis of CTLs and DCs}

CTLs were collected in media from individual wells and added to $15 \mathrm{~mL}$ conical tubes. Dendritic cells were dissociated from the wells using Cell Dissociation Buffer (Life Technologies, \#13151-014) and added to the same tube. The cells were centrifuged at $300 \mathrm{x}$ g for 5 mins and resuspended in $100 \mu \mathrm{l} \% \mathrm{BSA}$ in PBS. CD8a (APC, Biolegend, 100711) and PD1 (FITC, Biolegend, $135213)$ were added (1:100) in the cell suspension. The cells were incubated in the dark at room temperature for 15 mins. Reagent A fixation medium (Invitrogen, \#GAS001) was added to the cell suspension for 15 mins at room temperature. 5\% BSA in PBS was added to the cells and the suspension was centrifuged at $300 \mathrm{~g}$ for 5 mins. The cell pellets were resuspended in $100 \mu \mathrm{l}$ Reagent 
B permeabilization buffer (Invitrogen, GAS002). Primary antibodies IFN- $\gamma$ (PE, Biolegend, 505807) and IL-2 (Brilliant Violet 421, Biolegend, 503825) were added (1:100) in the cell suspension and incubated for 20 mins at room temperature in the dark. 5\% BSA diluted in PBS was added to the cell suspension and centrifuged at $300 \mathrm{~g}$ for $5 \mathrm{~min}$. The labeled CTLs were resuspended in 5\% BSA in PBS and the activation was analyzed using Canto-III flow cytometer. In a separate series of cultures, DCs were pulsed with $\mathrm{mTGO}^{\mathrm{CM}}$. The matured DCs were collected and immunostained with CD40 (APC, Biolegend, 124611), CD80 (BV421, Biolegend, 104725), CD86 (PE, Biolegend, 105105), CD11c (BV650, Biolegend, 117339), and I-AB (FITC, Biolegend, 114406). Labeled DCs were then analyzed using Canto-III flow cytometer and FlowJo data analysis program.

\section{Quantitative RT-PCR (qRT-PCR)}

RNA was isolated from either stomach tissue of iLgr5;GLI2A mice or xenografted tumor tissue using TRIzol Reagent (Molecular research Center, TR118) according to the manufacturer's instructions. The High Capacity cDNA Reverse Transcription Kit (Applied Biosystems) was used for cDNA synthesis of RNA following the manufacturer's protocol. For each sample $100 \mathrm{ng}$ of RNA was reverse transcribed to yield approximately $2 \mu \mathrm{g}$ total cDNA that was then used for the real-time PCR. Pre-designed Real-Time PCR assays were purchased for the following genes (Thermo Fisher, Applied Biosystems): Mouse (Mm) or human (Hs)specific GAPDH (Hs02786624_g1; Mm99999915_g1), PD-L1 (Mm03048248_m1, Hs00204257_m1), PTCH1 (Mm00436026_m1; Hs00181117_m1), PTCH2 (Mm00 436047_m1; Hs00184804_m1), GLI1 (Mm00494654_m1; Hs00171790_m1), HHIP(Mm00469580_m1; Hs0101015 m1), CD8 (-Mm01182107_g1), and GranzymeB (Mm0 1322702 m1). PCR amplifications were performed in a total volume of $20 \mu \mathrm{l}$, containing 20X TaqMan Expression Assay primers, 2X TaqMan Universal Master Mix (Applied Biosystems, TaqMan ${ }^{\circledR}$ Gene Expression Systems) and cDNA template. Each PCR amplification was performed in duplicate wells in a StepOne ${ }^{\mathrm{TM}}$ Real-Time PCR System (Applied Biosystems), using the following conditions: $50^{\circ} \mathrm{C} 2$ minutes, $95^{\circ} \mathrm{C} 10$ minutes, $95^{\circ} \mathrm{C} 15$ seconds (denature) and $60^{\circ} \mathrm{C} 1$ minute (anneal/extend) for 40 cycles. Fold change was calculated as: $\left(\mathrm{C}_{\mathrm{t}}-\mathrm{C}_{\mathrm{thigh}}\right)=$ $\mathrm{n}_{\text {target }}, 2^{\text {ntarget }} / 2^{\text {nHPRT }}=$ fold change where $\mathrm{C}_{\mathrm{t}}=$ threshold cycle. The results were expressed as average fold change in gene expression relative to control with GAPDH used as an internal control according to Livak and Schmittgen [37].

\section{Western blot}

Organoids were lysed in M-PER Mammalian Protein Extraction Reagent (Thermo Scientific, 78501) supplemented with protease inhibitors (Roche, 05892970
001) according to the manufacturer's protocol. Cell lysates were resuspended in $40 \mu \mathrm{l}$ Laemmli Loading Buffer containing $\beta$-mercaptoethanol (Bio-Rad Laboratories, 1610730) before western blot analysis. Samples were loaded onto 4-20\% Tris-Glycine Gradient Gels (Invitrogen) and run at 80 volts for approximately 3 hours and transferred to nitrocellulose membranes (Whatman Protran, $0.45 \mu \mathrm{M})$ at 105 volts for 1.5 hours at $4^{\circ} \mathrm{C}$. Membranes were blocked for 1 hour at room temperature using KPL Detector Block Solution (Kirkegaard \& Perry Laboratories, Inc., 718300). Membranes were incubated for 16 hours at $4{ }^{\circ} \mathrm{C}$ with either 1:100 dilution of rat antiPD-L1 (Novus Biologicals, NBP1-76769), or 1:2000 dilution of mouse anti-GAPDH (Millipore, MAB374) antibodies followed by 1 hour incubation with a 1:1000 dilution anti-mouse, or -rat Alexa Fluor 680 (Invitrogen). Blots were imaged using a scanning densitometer along with analysis software (Odyssey Infrared Imaging Software System) and the ratio of PD-L1/GAPDH was calculated using ImageJ.

\section{Drug assay in human tumor-derived organoids}

Human-derived gastric cancer organoids (huTGOs) were grown in 96 well plates and treated with either epirubicin (Selleckchem, S1223), oxaliplatin (Sigmaaldrich, 9512), or 5-Fluorouracil (Selleckchem, S1209) at concentrations of $0,0.5,1,5,10,50,100$ and $200 \mu \mathrm{M}$ or in combination with $5 \mu \mathrm{M}$ GANT61 (Stemcell Technologies, 73692) for 48 hours. After 48 hours, organoid proliferation was measured using MTS Assay (Promega, G3580). The absorbance was measured at 495 $\mathrm{nm}$, concentrations were transformed in logarithmic scale, and a best fit nonlinear dose-response curve was plotted using GraphPad Prism (GraphPad Software, San Diego, CA). huTGOs were also grown in 48 well tissue culture plates and treated with either DMSO, or, the combination of epirubicin, oxaliplatin, and 5-FU at their calculated IC50 concentration, or $5 \mu \mathrm{M}$ GANT61 plus a combination of epirubicin, oxaliplatin, and 5-FU at their calculated EC50 concentration. The organoids were harvested at different time points $(0,24,48$ and $72 \mathrm{hrs})$, resuspended in 5\% BSA in PBS and stained with calcein AM and Ethidium homodimer1 for $30 \mathrm{~min}$, room temperature at dark (ThermoFisher Scientific, L3224). Labeled cells were analyzed using Canto-III flow cytometer. The average ratio of live/dead cells were calculated using FlowJo and plotted as mean \pm SEM using GraphPad Prism software.

\section{Mouse xenograft assay}

Xenograft assays were performed by injecting organoids (huTGO2) subcutaneously in the right flank of NSG mice. Tumor dimensions were measured every 7 days. In mice transplanted with gastric cancer-derived organoids, once tumor volume reached 100 cubic millimeters (within 14 days of transplantation), animals 
were treated with either vehicle (PBS, i.p.), or GANT61 (50 mg/kg in PBS, i.p. every alternate days), or cisplatin ( $3 \mathrm{mg} / \mathrm{kg}$ in saline, i.p. 2 days/week, Selleckchem, S1166), or cisplatin plus GANT61 for 30 days. Tumor height (h), length (l) and width (w) were measured once/week using a caliper. Tumor volume was calculated using a published equation [38].

\section{Immunofluorescence}

Stomach tissues were fixed in 4\% paraformaldehyde for 16 hours, paraffin-embedded and sectioned at $5 \mu \mathrm{m}$. Tissue slides were deparaffinized and boiled in antigen citrate buffer (Vector Laboratories, H3300) for 10 minutes. Sections were then blocked with $20 \%$ donkey serum for 20 minutes, and immunostained with primary antibodies overnight at $4^{\circ} \mathrm{C}$, followed by incubation with secondary antibodies for 1 hour. Coverslips were mounted onto slides with Vectashield Mounting Medium (Vector Laboratories, H-1400). For whole mount staining, gastric organoids were fixed in 3.7\% formaldehyde for 15 minutes at room temperature, followed by washing in DPBS. Organoids were permeabilized with $0.5 \%$ Triton $\mathrm{X}-100$ for 20 minutes at room temperature. Organoids were then blocked with $2 \%$ donkey serum for $1 \mathrm{hr}$ and incubated with primary antibody overnight and washed in PBS containing $0.01 \%$ Triton-X 100. Secondary antibody incubation was also performed overnight in gastric organoids, and subsequently immunostained for cell nuclei using $10 \mu \mathrm{g} / \mathrm{mL}$ Hoechst. The following primary antibodies and dilutions were used: 1:100 human and mouse -specific rat anti PD-L1 (Novus Biologicals, NBP1-43262), 1:100 human and mouse-specific rabbit anti Gli-2 (Novus Biologicals, NBP2-23602), 1:100 human and mouse-specific rabbit anti PD-L1 (Novus Biologicals, NBP1-76769), 1:100 human-specific rabbit anti-Histone H1.0 (abcam, ab125027), 1:200 mouse alpha smooth muscle actin (SMA, Novus Biologicals, NBP222120) or 1:2000 dilution of goat anti-intrinsic factor (IF, a kind gift from Dr. David Alpers, Washington University School of Medicine in St. Louis). Both slides and whole mount organoids were imaged on a Zeiss LSM710 LIVE Duo Confocal Microscope.

\section{Immunohistochemistry}

Stomach tissues were fixed in $4 \%$ paraformaldehyde for 15 minutes, paraffin embedded, and $5 \mu \mathrm{m}$ sections were cut. After deparaffinization, antigen retrieval was performed by heating the slides for 10 minutes at $100^{\circ} \mathrm{C}$ in $0.01 \mathrm{M}$ sodium citrate buffer (Antigen Unmasking Solution, Vector Laboratories, Burlingame, CA). Endogenous peroxidase activity was then blocked by incubating slides in $0.3 \%$ hydrogen peroxide/methanol for 20 minutes followed by 20 minutes incubation with $20 \%$ goat serum. Slides were incubated with either 1:100 dilution of PCNA antibody
(Novus Biologicals, NB100-456), PD-L1 antibody (Novus Biologicals, NBP1-76769) or Ki67 (Fisher scientific, Rm-9106RQ) overnight at $4^{\circ} \mathrm{C}$. Slides were then incubated with biotinylated anti-rabbit $\mathrm{IgG}$ secondary antibody for 30 minutes followed by additional 30 minute incubation with $\mathrm{ABC}$ reagent (Vectastain $\mathrm{ABC}$ kit; Vector Laboratories, Burlingame, CA). The color was developed with 3,3'-diaminobenzidine (DAB) using the DAB Substrate Kit (Vector Laboratories, Burlingame, CA) and counterstained with hematoxylin (Fisher Scientific Company, 245-653), dehydrated and mounted with Permount (Fisher Scientific company, SP15-100).

Paraffin embeded stomach tissues were also labeled for PCNA and CD8 antigens using ImmPRESS reagents. Briefly, after the blocking of endogenous peroxidase activity, the slides were blocked with $2.5 \%$ normal horse serum. The slides were then incubated with 1:2000 dilution of PCNA antibody (Novus Biologicals, NB100456) overnight at $4^{\circ} \mathrm{C}$, followed by 30 minute incubation with anti-rabbit ImmPRESS Ig (Vector Lab, MP-7401) and color was developed using peroxidase substrate solution (Vector lab, SK-4605). After washing, slides were blocked for a second time with $2.5 \%$ normal goat serum, and incubated with 1:200 dilution of CD8 antibody (Novus Biologicals, NBP1-49045) overnight at $4^{\circ} \mathrm{C}$, followed by 30 minute incubation with anti-rat ImmPRESS Ig (Vector Lab, MP-7444) and color developed using peroxidase substrate solution (Vector lab, SK-4105). After completion of immunohistochemical staining, slides were counterstained with Methyl Green (Vector lab, H-3402) and mounted with Permount (Fisher Scientific Company, SP15-100).

\section{Statistical analyses}

The significance of the results was tested by oneway ANOVA or student's $t$-test using commercially available software (GraphPad Prism Software, San Diego, CA). A $P$ value $<0.05$ was considered as the level of significance.

\section{Abbreviations}

PD-L1: Programmed cell death - ligand 1; PD-1: Programmed cell death protein - 1; PD-L1Ab: Cytotoxic T Lymphocytes; CTL: PD-L1 neutralizing antibody; CD8: Cluster of differentiation 8; Hh: Hedgehog; DC: Dendritic cells; $i L g r 5$; GLI2A: triple-transgenic Lgr5EGFP-IRES-CreERT2; R26-LSL-rtTA-IRES-EGFP; tetO-GLI2A mouse; PBS: Phosphate buffered saline; DPBS: Dulbecco'sPhosphate buffered saline; qRT PCR: Quantitative reverse transcription polymerase chain reaction; mTGO: Mouse tumor derived gastric organoids; mGO: Mouse gastric organoids; huTGO: Human tumor derived gastric organoids; EDTA: HBSS: 
Hank's Balanced Salt Solution; DMEM: Dulbecco's Modified Eagle's medium; EGF: Epidermal growth factor; FGF10: Fibroblast growth factor 10; FCS: Fetal Calf Serum; rmGMCSF: Recombinant mouse granulocyte-macrophage colony stimulating factor; CM: Conditioned media; RPMI: Roswell park memorial institute medium; BSA: Bovine serum albumin; 5FU: 5 Fluorouracil; PCNA: Proliferating cell nuclear antigen; IF: Intrinsic Factor.

\section{Author contributions}

Jayati Chakrabarti: 1) the conception and design of the study, acquisition of data, or analysis and interpretation of data;2) drafting of the article or revising it for important content; and 3) final approval of the version to be published. Loryn Holokai: 1) the conception and design of the study interpretation of data; 2) drafting of the article or revising it for important content; and 3) final approval of the version to be published. LiJyun Syu: 1) the conception and design of the study; 2) drafting of the article or revising it for important content; and 3) final approval of the version to be published. Nina G. Steele: 1) the conception and design of the study, acquisition of data, or analysis and interpretation of data; 2) drafting of the article or revising it for important content; and 3) final approval of the version to be published. Julie Chang: 1) acquisition of data, or analysis and interpretation of data; 2) drafting of the article or revising it for important content; and 3) final approval of the version to be published. Jiang Wang: 1) the conception and design of the study, 2) final approval of the version to be published. Syed Ahmed: 1) the conception and design of the study, 2) drafting of the article or revising it for important content; and 3) final approval of the version to be published. Andrzej Dlugosz: 1) the conception and design of the study, interpretation of data; 2) drafting of the article or revising it for important content; and 3) final approval of the version to be published. Yana Zavros: 1) the conception and design of the study, acquisition of data, or analysis and interpretation of data; 2) drafting of the article or revising it for important content; and 3) final approval of the version to be published. Ensuring that all authors have agreed 1) to be authors and to be listed in the order specified by the submitting author; 2) to the manuscript's content; and 3) to its submission to the journal.

\section{ACKNOWLEDGMENTS}

We would like to acknowledge the assistance of Chet Closson (Live Microscopy Core, University of Cincinnati). We thank Lisa McMillin (Cincinnati Children's Hospital Medical Center, Pathology Research Core) for her assistance with organoid embedding and processing. We thank Kathy McClinchey and the McClinchey Histology Lab Inc.. Finally, we sincerely thank the patients that consented to donate tissue for the development of the gastric organoids. Without their willingness to participate in the study, this work would not be possible.

\section{CONFLICTS OF INTEREST}

The authors have nothing to disclose.

\section{FUNDING}

This work was supported by NIH (NIDDK) 2 R01 DK083402-06A1 grant and College of Medicine Bridge Funding Program (YZ). This project was supported in part by PHS Grant P30 DK078392 (Integrative Morphology Core) of the Digestive Diseases Research Core Center in Cincinnati.

\section{REFERENCES}

1. Ahmad SA, Xia BT, Bailey CE, Abbott DE, Helmink BA, Daly MC, Thota R, Schlegal C, Winer LK, Ahmad SA, Al Humaidi AH, Parikh AA. An update on gastric cancer. Curr Probl Surg. 2016; 53:449-90.

2. Sitarz R, Skierucha M, Mielko J, Offerhaus GJA, Maciejewski R, Polkowski WP. Gastric cancer: epidemiology, prevention, classification, and treatment. Cancer Manag Res. 2018; 10:239-48.

3. Stock M, Otto F. Gene deregulation in gastric cancer. Gene. 2005; 360:1-19.

4. Sriamporn S, Setiawan V, Pisani P, Suwanrungruang K, Sirijaichingkul S, Mairiang P, Parkin DM. Gastric Cancer: the Roles of Diet, Alcohol Drinking, Smoking and Helicobacter pylori in Northeastern Thailand. Asian Pac J Cancer Prev. 2002; 3:345-52.

5. Janowitz T, Thuss-Patience P, Marshall A, Kang JH, Connell C, Cook N, Dunn J, Park SH, Ford H. Chemotherapy vs supportive care alone for relapsed gastric, gastroesophageal junction, and oesophageal adenocarcinoma: a meta-analysis of patient-level data. Br J Cancer. 2016; 114:381-7.

6. Thuss-Patience PC, Kretzschmar A, Bichev D, Deist T, Hinke A, Breithaupt K, Dogan Y, Gebauer B, Schumacher G, Reichardt P. Survival advantage for irinotecan versus best supportive care as second-line chemotherapy in gastric cancer--a randomised phase III study of the Arbeitsgemeinschaft Internistische Onkologie (AIO). Eur J Cancer. 2011; 47:2306-14.

7. Smyth E, Thuss-Patience PC. Immune Checkpoint Inhibition in Gastro-Oesophageal Cancer. Oncol Res Treat. 2018; 41:272-80.

8. Ahmadzadeh M, Johnson LA, Heemskerk B, Wunderlich JR, Dudley ME, White DE, Rosenberg SA. Tumor antigen- 
specific CD8 T cells infiltrating the tumor express high levels of PD-1 and are functionally impaired. Blood. 2009; 114:1537-44.

9. Chen X, Fosco D, Kline DE, Meng L, Nishi S, Savage PA, Kline J. PD-1 regulates extrathymic regulatory T-cell differentiation. Eur J Immunol. 2014; 44:2603-16.

10. Reissfelder C, Stamova S, Gossmann C, Braun M, Bonertz A, Walliczek U, Grimm M, Rahbari NN, Koch M, Saadati M, Benner A, Büchler MW, Jäger D, et al. Tumor-specific cytotoxic $\mathrm{T}$ lymphocyte activity determines colorectal cancer patient prognosis. J Clin Invest. 2015; 125:739-51.

11. Yang Z, Lei Y, Chen C, Ren H, Shi T. Roles of the programmed cell death $1, \mathrm{~T}$ cell immunoglobulin mucin-3, and cluster of differentiation 288 pathways in the low reactivity of invariant natural killer $\mathrm{T}$ cells after chronic hepatitis B virus infection. Arch Virol. 2015; 160:2535-45.

12. Ingham PW, McMahon AP. Hedgehog signaling in animal development: paradigms and principles. Genes Dev. 2001; 15:3059-87.

13. Saqui-Salces M, Merchant JL. Hedgehog signaling and gastrointestinal cancer. Biochim Biophys Acta. 2010; 1803:786-95.

14. Cancer Genome Atlas Research N. Comprehensive molecular characterization of gastric adenocarcinoma. Nature. 2014; 513:202-9.

15. Onishi H, Fujimura A, Oyama Y, Yamasaki A, Imaizumi A, Kawamoto M, Katano M, Umebayashi M, Morisaki T. Hedgehog signaling regulates PDL-1 expression in cancer cells to induce anti-tumor activity by activated lymphocytes. Cell Immunol. 2016; 310:199-204.

16. Syu LJ, Zhao X, Zhang Y, Grachtchouk M, Demitrack E, Ermilov A, Wilbert DM, Zheng X, Kaatz A, Greenson JK, Gumucio DL, Merchant JL, di Magliano MP, et al. Invasive mouse gastric adenocarcinomas arising from Lgr5+ stem cells are dependent on crosstalk between the Hedgehog/ GLI2 and mTOR pathways. Oncotarget. 2016; 7:10255-70. https://doi.org/10.18632/oncotarget.7182.

17. Leushacke M, Tan SH, Wong A, Swathi Y, Hajamohideen A, Tan LT, Goh J, Wong E, Denil SLIJ, Murakami K, Barker N. Lgr5-expressing chief cells drive epithelial regeneration and cancer in the oxyntic stomach. Nat Cell Biol. 2017; 19:774-86.

18. Xiao C, Feng R, Engevik AC, Martin JR, Tritschler JA, Schumacher M, Koncar R, Roland J, Nam KT, Goldenring JR, Zavros Y. Sonic Hedgehog contributes to gastric mucosal restitution after injury. Lab Invest. 2013; 93:96-111.

19. Engevik AC, Feng R, Yang L, Zavros Y. The Acid-secreting parietal cell as an endocrine source of sonic hedgehog during gastric repair. Endocrinology. 2013; 154:4627-39.

20. Szczepny A, Rogers S, Jayasekara WSN, Park K, McCloy RA, Cochrane CR, Ganju V, Cooper WA, Sage J, Peacock CD, Cain JE, Burgess A, Watkins DN. The role of canonical and non-canonical Hedgehog signaling in tumor progression in a mouse model of small cell lung cancer. Oncogene. 2017; 36:5544-50.
21. Bertaux-Skeirik N, Centeno J, Gao J, Gabre J, Zavros Y. Oncogenic Transformation of Human-Derived Gastric Organoids. Methods Mol Biol. 2016 Aug 19. https://doi. org/10.1007/7651_2016_4. [Epub ahead of print].

22. Zou W, Chen L. Inhibitory B7-family molecules in the tumour microenvironment. Nat Rev Immunol. 2008; 8:467-77.

23. Parsa AT, Waldron JS, Panner A, Crane CA, Parney IF, Barry JJ, Cachola KE, Murray JC, Tihan T, Jensen MC, Mischel PS, Stokoe D, Pieper RO. Loss of tumor suppressor PTEN function increases B7-H1 expression and immunoresistance in glioma. Nat Med. 2007; 13:84-8.

24. Akbay EA, Koyama S, Carretero J, Altabef A, Tchaicha JH, Christensen CL, Mikse OR, Cherniack AD, Beauchamp EM, Pugh TJ, Wilkerson MD, Fecci PE, Butaney M, et al. Activation of the PD-1 pathway contributes to immune escape in EGFR-driven lung tumors. Cancer Discov. 2013; 3:1355-63.

25. Mittendorf EA, Philips AV, Meric-Bernstam F, Qiao N, Wu Y, Harrington S, Su X, Wang Y, Gonzalez-Angulo AM, Akcakanat A, Chawla A, Curran M, Hwu P, et al. PD-L1 expression in triple-negative breast cancer. Cancer Immunol Res. 2014; 2:361-70.

26. Dong H, Zhu G, Tamada K, Chen L. B7-H1, a third member of the B7 family, co-stimulates T-cell proliferation and interleukin-10 secretion. Nat Med. 1999; 5:1365-9.

27. Gu L, Chen M, Guo D, Zhu H, Zhang W, Pan J, Zhong X, Li X, Qian H, Wang X. PD-L1 and gastric cancer prognosis: A systematic review and meta-analysis. PLoS One. 2017; 12:e0182692.

28. Boger C, Behrens HM, Mathiak M, Kruger S, Kalthoff H, Rocken C. PD-L1 is an independent prognostic predictor in gastric cancer of Western patients. Oncotarget. 2016; 7:24269-83. https://doi.org/10.18632/oncotarget.8169.

29. Chang H, Jung WY, Kang Y, Lee H, Kim A, Kim HK, Shin BK, Kim BH. Programmed death-ligand 1 expression in gastric adenocarcinoma is a poor prognostic factor in a high $\mathrm{CD} 8+$ tumor infiltrating lymphocytes group. Oncotarget. 2016; 7:80426-34. https://doi.org/10.18632/ oncotarget.12603.

30. Steinman RM. The dendritic cell system and its role in immunogenicity. Annu Rev Immunol. 1991; 9:271-96.

31. Matsueda S, Graham DY. Immunotherapy in gastric cancer. World J Gastroenterol. 2014; 20:1657-66.

32. Kono K, Rongcun Y, Charo J, Ichihara F, Celis E, Sette A, Appella E, Sekikawa T, Matsumoto Y, Kiessling R. Identification of HER2/neu-derived peptide epitopes recognized by gastric cancer-specific cytotoxic $\mathrm{T}$ lymphocytes. Int J Cancer. 1998; 78:202-8.

33. Song X, Yan L, Lu C, Zhang C, Zhu F, Yang J, Jing H, Zhang Y, Qiao J, Guo H. Activation of hedgehog signaling and its association with cisplatin resistance in ovarian epithelial tumors. Oncol Lett. 2018; 15:5569-76.

34. Olive KP, Jacobetz MA, Davidson CJ, Gopinathan A, McIntyre D, Honess D, Madhu B, Goldgraben MA, Caldwell ME, Allard D, Frese KK, Denicola G, Feig C, 
et al. Inhibition of Hedgehog signaling enhances delivery of chemotherapy in a mouse model of pancreatic cancer. Science. 2009; 324:1457-61.

35. Schumacher MA, Aihara E, Feng R, Engevik A, Shroyer NF, Ottemann KM, Worrell RT, Montrose MH, Shivdasani RA, Zavros Y. The use of murine-derived fundic organoids in studies of gastric physiology. J Physiol. 2015; 593:1809-27.

36. Wang W, Li J, Wu K, Azhati B, Rexiati M. Culture and Identification of Mouse Bone Marrow-Derived Dendritic Cells and Their Capability to Induce $\mathrm{T}$ Lymphocyte Proliferation. Med Sci Monit. 2016; 22:244-50.
37. Livak K, Schmittgen T. Analysis of relative gene expression data using real-time quantitative PCR and the 2(-Delta Delta C(T)) Method. Methods. 2001; 25:402-8.

38. Tomayko MM, Reynolds CP. Determination of subcutaneous tumor size in athymic (nude) mice. Cancer Chemother Pharmacol. 1989; 24:148-54. 